\title{
EchoGéo
}

$37 \mid 2016$

Femmes et migrations : celles qui restent

\section{Émigration masculine et développement touristique en versant sud du massif des Annapurna (Népal) : les femmes à la manœuvre}

Pierre Dérioz, Pranil Upadhayaya, Maud Loireau, Philippe Bachimon, Justine Le Noac'h et Mauve Létang

\section{OpenEdition}

\section{Journals}

Édition électronique

URL : https://journals.openedition.org/echogeo/14724

DOI : $10.4000 /$ echogeo. 14724

ISSN : 1963-1197

Éditeur

Pôle de recherche pour l'organisation et la diffusion de l'information géographique (CNRS UMR 8586)

Référence électronique

Pierre Dérioz, Pranil Upadhayaya, Maud Loireau, Philippe Bachimon, Justine Le Noac'h et Mauve Létang, «Émigration masculine et développement touristique en versant sud du massif des Annapurna (Népal) : les femmes à la manœuvre », EchoGéo [En ligne], 37 | 2016, mis en ligne le 31 août 2021, consulté le 31 août 2021. URL : http://journals.openedition.org/echogeo/14724 ; DOI : https:// doi.org/10.4000/echogeo.14724

Ce document a été généré automatiquement le 31 août 2021.

EchoGéo est mis à disposition selon les termes de la licence Creative Commons Attribution - Pas d'Utilisation Commerciale - Pas de Modification 4.0 International (CC BY-NC-ND) 


\title{
Émigration masculine et développement touristique en versant sud du massif des Annapurna (Népal) : les femmes à la manœuvre
}

\author{
Pierre Dérioz, Pranil Upadhayaya, Maud Loireau, Philippe Bachimon, \\ Justine Le Noac'h et Mauve Létang
}

\section{Introduction}

1 En dépit de l'inscription dans des textes de loi comme le $11^{\mathrm{e}}$ amendement du Code Civil de mars 2002 (Women's Bill) ou la Loi sur l'Égalité des Genres (28 septembre 2006) d'un certain nombre de dispositions en faveur de l'égalité hommes-femmes, le statut des femmes népalaises reste dans les faits largement soumis aux dispositions restrictives et aux normes patriarcales héritées de la tradition hindoue. En net décalage avec les efforts de réforme (Giri, 2012), elles se traduisent par une discrimination systématique des femmes qui concerne tous les aspects de leur vie au plan social comme aux plans économique et politique (Subedi, 1997 ; Acharya, 2003 et 2007 ; Mahat, 2003 ; Bennett, 2005 ; Kaspar, 2005, p. 21-24 ; Shakya, 2014 ; Bhattarai, 2014). Étroitement contraintes par les rôles successifs qui leur sont assignés au sein de la famille (Luitel, 2001), les femmes se trouvent placées en position de subordination et de domination par les hommes - père, mari, fils -, en vertu des pratiques coutumières qui régissent le mariage (mariages souvent précoces, arrangés par les familles, obligations liées à la dot et à la patrilocalité, attentes en matière de fertilité et préférence pour les enfants mâles...) et des impératifs rituels de pureté auxquels elles sont astreintes (isolement pendant les menstruations, en particulier). Les femmes sont confinées dans les tâches ménagères, la prise en charge des enfants et les travaux agricoles, sans beaucoup d'opportunités d'accès à un travail salarié, ni beaucoup de poids dans les processus de 
décision, tant domestiques que collectifs (Acharya et al., 2010). Leurs droits en matière de propriété (notamment celle de la terre, cf Campus, 2016) et d'héritage sont limités et surtout peu respectés, leur accès à l'éducation scolaire est plus réduit que celui des garçons (au recensement de 2011, 42,2 \% de femmes étaient illettrées, contre $24 \%$ des hommes). De très nombreux cas de violence à leur encontre sont encore relevés.

2 Même si des évolutions positives peuvent être observées dans certains domaines (Pokharel, 2010; Shakya, 2014), la question des rapports de genre apparait ainsi particulièrement prégnante au Népal. De nombreux auteurs la traduisent aujourd'hui en termes "d'empowerment » des femmes, anglicisme dont le succès à partir des années 1990, notamment auprès d'institutions internationales comme les Nations Unies ou la Banque Mondiale, a quelque peu éclipsé d'autres termes dont il ne recouvre pourtant pas nécessairement la signification exacte: «insertion» ou « autonomisation » ne le traduisent en français que de manière approximative pour A. Calvès (2009), et N. Kabeer (2008, p. 14-15) le trouve en définitive plus proche des premiers usages par les mouvements féministes du mot «libération » que du sens que revêt le terme " émancipation », que d'autres auteurs pourtant (Pearson, 2004 ; Moser, 2007) regardent comme assez équivalent à celui d'empowerment. La principale critique formulée à l'encontre de cette notion réside dans le fait que son intégration spectaculaire dans la terminologie des grandes agences internationales s'est accompagnée d'une perte de substance, sinon même d'un dévoiement, par rapport aux approches initiales des activistes et des chercheurs, l'absence de définition claire favorisant un consensus vague, peu subversif et peu opérationnel (Parpart et al., 2002 ; Parpart, 2002 ; Calvès, 2014), plutôt axé sur la promotion de formes d'entrepreneuriat individuel (Hofmann et Marius-Gnanou, 2007).

3 Le choix d'utiliser le terme d'empowerment dans cet article s'inscrit donc dans le courant qui en «rejette[nt] la version individualiste, dépolitisée, verticale et instrumentale » (Calvès, 2009) et s'efforce de lui restituer sa pleine signification, multidimensionnelle, contextualisée, collective et transcalaire. Pour N. Kabeer (1999), la mesure de l'empowerment se place ainsi dans une perspective dynamique qui interconnecte étroitement trois dimensions du pouvoir, l'accès aux ressources (matérielles, mais aussi intellectuelles, techniques, culturelles...) en tant que pré-requis, la capacité d'initiative (agency) en tant que processus, et les résultats obtenus (achievements). Les deux premières réunies, ressources et capacité d'initiative, soustendent, selon N. Kabeer, la notion de « capabilités » (capabilities) développée par A. Sen (2009). Une autre approche, plus analytique, relève six dimensions différentes en interaction (économique, socioculturelle, familiale-relationnelle, juridique, politique et psychologique), et recommande leur prise en compte à la fois aux échelles élémentaires (individus, ménages), intermédiaires (communautés) et plus globales (Malhotra et al., 2003). Une troisième approche (citée in Louargant et Perrin-Bensahel, 2007), qui recoupe partiellement les deux premières, identifie cinq niveaux hiérarchisés d'empowerment - bien-être, accès, conscientisation, participation, contrôle. Trois points clés se dégagent de ce tour d'horizon rapide, qui ont ici particulièrement balisé notre recherche :

- envisagé au plan individuel comme au plan collectif, l'empowerment articule aspects matériels, relations entre acteurs et systèmes de représentation; c'est à ces trois niveaux qu'il doit donc être observé ;

- en tant que processus de changement susceptible d'affecter aussi bien des éléments 
formels (institutions, lois...) qu'informels (pratiques, normes sociales...) (Rao \& Kelleher, 2005, p.60-61), il peut relever simultanément de l'action politique de mouvements revendicatifs à différentes échelles, d'ensembles de mesures et de dispositifs émanant de l'État ou d'instances internationales de différente nature (topdown), de démarches collectives explicites d'échelle plus locale (bottom-up), mais aussi des effets cumulatifs du déploiement des stratégies adaptatives de chaque acteur ou groupe d'acteurs ;

- le point précédent, enfin, implique la double inscription de l'observation de ce processus de changement dans l'appréhension des phénomènes globaux et dans celle des spécificités propres à chaque contexte territorial (Rai et al., 2007).

Cette insistance sur les éléments de contexte nuance fréquemment le tableau relativement sombre du statut des femmes népalaises que dressent les chercheurs, qui le modulent en fonction de critères socio-économiques (revenus des ménages, niveau d'éducation), ethniques et géographiques. Au nombre des facteurs dont les effets ont été fréquemment examinés se trouve en particulier le phénomène des migrations pour l'emploi, qui comporte d'ailleurs à la fois une dimension globale - elles correspondent à des flux nationaux et transnationaux - et une dimension locale - elles connaissent une intensité assez variable selon les régions considérées. L'émigration internationale de travail constitue en effet au Népal un phénomène ancien (Thieme, 2006) et de grande ampleur (Aubriot, Bruslé, 2012 ; Gurung, 2012), dont les derniers recensements (1991, 2001, 2011) montrent que l'intensité s'est encore accrue - $6,8 \%$ de la population népalaise totale était «absente» en $2011^{1}$, contre seulement 3,3\% en 2001. Dans la mesure où cette émigration demeure très majoritairement masculine ${ }^{2}$, à plus de $80 \%$ depuis les années 1950 (87,6\% en 2011), et compte tenu des durées d'absence des migrants - généralement plusieurs années -, elle se traduit localement par un déséquilibre durable dans les sex-ratios et une nette féminisation des sociétés locales, lisible dans le nombre croissant des foyers dont la personne référente est une femme (un quart de l'ensemble des foyers en 201133). De nombreux chercheurs (Kaspar, 2005 ; Gartaula, 2007 ; Gartaula et al., 2010 ; Thieme et al., 2011 ; Maharjan et al., 2012) ont donc cherché à déterminer si l'absence prolongée de nombreux hommes offrait aux femmes qui restaient au pays des opportunités d'autonomisation et de redéfinition de leur place au sein du ménage comme au sein des communautés, et jusqu'à quel point les changements observés pouvaient être considérés comme définitifs (i.e. s'ils se prolongeaient au-delà des retour des migrants). Bien que les travaux sur ce thème ne soient encore pas très nombreux, ceux réalisés sur le Népal s'inscrivent dans un questionnement qui a également été appliqué dans d'autres espaces, de l'Egypte (Brink, 1991 ; Louhichi, 1997) et du Mozambique (Yabiku et al., 2010) au Maroc (De Haas et Van Rooij, 2010) et au Sénégal (Mondain et al., 2012).

5 Situé en versant sud du Massif des Annapurna, le terrain d'étude évoqué dans cet article présente lui aussi un fort taux d'émigration masculine à l'étranger et un très net déséquilibre hommes-femmes, et à ce double titre se prête tout à fait à l'exploration de cette problématique. Mais ce secteur de piémont et de montagne proche de Pokhara, la seconde ville du pays, qui regroupe plusieurs itinéraires de trekking très prisés des touristes - dont le fameux trek « $\mathrm{ABC}$ » (Annapurna Base Camp) -, présente aussi la particularité d'être engagé dans un processus de développement touristique, plus ou moins ancien et intense selon les vallées et les villages. Là encore, un nombre croissant de travaux s'interrogent depuis une vingtaine d'années sur les opportunités d'empowerment qu'offrent aux femmes des pays du Sud les activités liées au tourisme, 
ou tout au moins à certaines formes de tourisme (écotourisme, tourisme communautaire, tourisme solidaire...), prenant acte du fait que le tourisme a souvent été regardé comme un outil des politiques de lutte contre la pauvreté («pro-poor tourism ", cf Ashley et al., 2000; Harrison, 2008) dont certaines se préoccupaient spécifiquement de la place des femmes (Scheyvens, 2000 ; Lama, 2000 ; Ateljevic, 2008 ; Ferguson, 2011). Des études critiques des apports et des limites, en matière d'empowerment, des activités touristiques en général et de ces politiques en particulier, ont été conduites dans des contextes assez différents, plus souvent ruraux (Swain, 1989; Tizza, 2012; Tiekoura, 2012; Pabion-Mouriès, 2012 ; Mrema, 2015) qu'urbains (Borghi, 2003 ; Zahedi, 2010), y compris au Népal, où K. Shakya (2014, p. 258) souligne à l'échelle du pays dans son entier la part prise par le secteur touristique dans l'engagement conscient ${ }^{4}$ des femmes dans les activités commerciales et de service.

6 C'est donc autour de la conjonction d'un double faisceau de facteurs potentiellement favorables à l'empowerment des femmes, forte émigration masculine d'une part et développement touristique sous diverses formes d'autre part, que s'articule la problématique de cet article. Après avoir rapidement présenté ces deux ensembles de facteurs (partie 1), nous nous efforcerons d'analyser comment les femmes se sont retrouvées de facto "à la manœuvre » face aux opportunités offertes par la venue des touristes, dans un contexte marqué simultanément par l'absence prolongée de nombreux hommes, et par le jeu interactif entre politiques volontaristes de développement touristique et expansion spontanée du secteur portée par des opérateurs privés (partie 2). Nous faisons ici l'hypothèse que la combinaison de ces facteurs a joué de manière positive sur la prise d'autonomie des femmes, leur accès aux ressources, aux compétences et aux responsabilités, et plus globalement la valorisation de leur identité culturelle et de leur image. Mais les limites de ce processus sont également envisagées (partie 3), à commencer par celles que lui impose l'ampleur des inégalités socio-économiques préexistantes : au sein de ces sociétés montagnardes où la diversité ethnique interfère avec le poids persistant du système de castes, les femmes, en effet, ne disposent pas toutes au départ des mêmes atouts pour se saisir de ces opportunités d'empowerment.

7 De manière un peu paradoxale, le matériau mobilisé pour cet article est issu d'une recherche qui n'est directement centrée ni sur les questions de genre ni sur celles liées aux migrations : le programme ANR AQAPA ${ }^{5}$ tente avant tout de décrire et d'interpréter les dynamiques territoriales et paysagères qui affectent des espaces ruraux montagnards en Asie occupés par des minorités ethniques, en prêtant une attention particulière aux effets des processus croisés de mise en tourisme et de patrimonialisation (paysages, milieux naturels, cultures locales,) qui s'y manifestent depuis plus ou moins longtemps. Le poids des phénomènes migratoires s'est donc plutôt imposé comme l'un des principaux déterminants pour la compréhension des situations locales analysées, et la place éminente occupée par les femmes dans les activités touristiques comme l'un des constats majeurs issus du travail de terrain. Ce dernier s'est déroulé dans le cadre de deux missions collectives (novembre 2014 et avril 2016) et de deux stages de Master $^{6}$, qui ont permis la réalisation de quelques 135 entretiens de type semi-directif ${ }^{7}$ avec des acteurs locaux (tableau 1), dont les enseignements ont été confrontés avec les informations produites à l'occasion des six groupes de discussion organisés (deux en contexte villageois, et quatre avec des opérateurs touristiques), et celles recueillies au fil du parcours à pied de l'aire d'étude. 
Tableau 1 - types d'acteurs interrogés (novembre 2014, avril-juin 2015, avril-mai 2016)

\begin{tabular}{|l|l|l|l|}
\hline & Femmes & Hommes & Total \\
\hline hôteliers (lodges/hôtels) & 5 & 7 & 12 \\
\hline employé sect. tourisme & 1 & 1 & 2 \\
\hline restaurateur & 1 & 2 & 3 \\
\hline opérateur de homestay (hébergement chez l'habitant) & 15 & 4 & 19 \\
\hline directeur agence trekking & 2 & 15 & 17 \\
\hline guide, acc. APN & 3 & 6 & 9 \\
\hline villageois / agriculteurs & 5 & 2 & 7 \\
\hline gestionnaire environnement & & 3 & 3 \\
\hline responsable local & 59 & 76 & 14 \\
\hline chef d'entreprise (hors tourisme) & 5 & 2 & 3 \\
\hline écolier / étudiant & & 2 & 2 \\
\hline enseignant & 3 & 2 & 2 \\
\hline travailleur émigré & & 18 & 39 \\
\hline touriste / trekkeur & & 35 \\
\hline Total & & 3 & 3 \\
\hline
\end{tabular}

De durée variable (20 minutes à 2h30), ces entretiens composent un échantillon qui s'est construit de manière itérative à partir d'une entrée principale autour des activités touristiques, et associe des personnes-ressource ciblées en amont et des rencontres de terrain imprévues. Le tableau comporte une part d'approximation, dans la mesure où certains acteurs ont plusieurs rôles (opératrice de homestay et présidente du « Groupe des Mères » local, par exemple), et où certains entretiens se sont déroulés avec plusieurs personnes simultanément (mari et femme, par exemple).

\section{Un espace rural montagnard entre migration de travail et développement touristique}

\section{Un double gradient : altitudes et proximité à la ville}

Deux gradients spatiaux peuvent guider la présentation du terrain sur lequel se déroule cette étude, intégralement compris au sein de l'Annapurna Conservation Area Project $(\mathrm{ACAP})^{8}$. Le premier est un gradient altitudinal globalement méridien, pour cet ensemble de onze $\mathrm{VDC}^{9}$ en versant sud du massif des Annapurna (carte 1), structuré par trois grandes vallées méridiennes - d'ouest en est, Modi Khola, Mardi Khola et Seti 
Nadi, appartenant toutes au bassin versant de la Kali Gandaki. L'habitat et l'essentiel des activités traditionnelles s'y étagent entre $1000 \mathrm{~m}$ et $4000 \mathrm{~m}$ environ, dans cette partie inférieure que J. Smadja (2003, p. 60) qualifie de "moyenne montagne», audessus de laquelle se déploie la "haute montagne", dont les crêtes et les sommets culminent entre 6000 et $8000 \mathrm{~m}$. Largement dominantes, les activités agro-pastorales, à finalité encore principalement vivrière, se répartissent en fonction de l'altitude : il s'agit pour l'essentiel de petites unités familiales de polyculture-élevage de montagne, combinant dans l'étage des villages (1000-2000 m d'altitude) rizières en terrasse irriguée plutôt en bas de versant (khet) et cultures sèches plus diversifiées au-dessus (bari), en particulier millet, maïs et pommes de terre. L'élevage, en partie transhumant, se déploie sur toute la gamme altitudinale, des espaces non cultivés et des chaumes à proximité des villages jusqu'aux pelouses d'altitude en passant par de nombreuses clairières intermédiaires au sein de versants forestiers longs et raides. Les quelques formes d'intensification (tracteurs, serres...), de diversification (plantations coopératives de thé à Lwang) ou de spécialisation à visée commerciale (élevage bovin, Tuse) peuvent être observées dans les plus gros villages, installés sur les terrasses alluviales les plus larges en partie aval des vallées.

\section{Illustration 1- Situation des 11 Village Development Committee du périmètre d'étude}

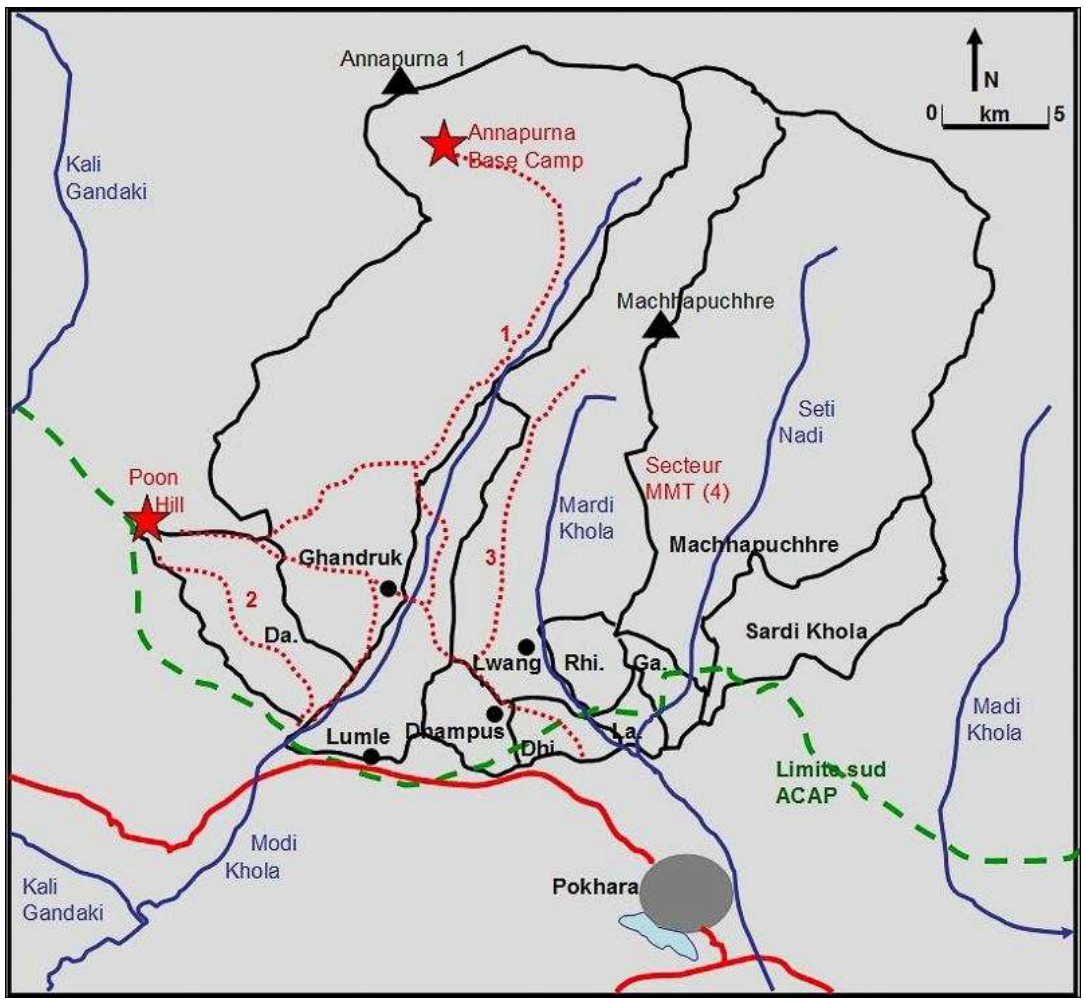

Da. : Dangsing / Dhi. : Dhital / Ga. : Ghachok / La. : Lahachok / Rhi. : Rhiban - 1 : Trek ABC / 2 : Balcon des Annapurna / 3 : Mardi Himal Trek / 4 : Machhapuchhre Model Trek.

lignes bleues : rivières, lignes rouges : routes principales, lignes rouges pointillées : itinéraires de trekking, tireté vert : limite de la zone ACAP, lignes noires : limites de VDC.

9 Le deuxième gradient renvoie à la plus ou moins grande proximité par rapport à l'agglomération en très forte croissance de Pokhara (Muzzini et Aparicio, 2013) et ses services (médicaux, scolaires...), ses commerces ou ses emplois, proximité temps plutôt que proximité distance compte tenu des obstacles que le relief et les rivières opposent à 
la circulation. Mais le temps n'est plus où R. Matthiessen (2003, p. 30 / 1 ère éd. 1978) pouvait écrire "There are no roads west of Pokhara, which is the last outpost of the moderne world; in one days walk we are a century away ${ }^{10}$." Le déploiement rapide depuis une petite dizaine d'années d'un réseau de pistes (plus ou moins carrossables) dont les saignées poussiéreuses marquent les paysages, a amorcé le désenclavement de ces vallées et facilité la circulation des hommes et des marchandises, entraînant de nouvelles pratiques (comme des mobilités hebdomadaires entre Pokhara et les villages de montagne, en bus, en jeep ou en moto) et induisant d'autres mutations paysagères (par exemple l'effet d'une plus grande facilité de transport des matériaux sur les nouvelles constructions).

\section{Formes et intensités du développement touristique}

10 Le système touristique régional est centré sur Pokhara, point de passage obligé des touristes, ville-siège de toutes les agences et centre organisateur des circuits et des activités : l'offre sur le terrain, liée à des initiatives locales, concerne seulement, sauf exception, les fonctions d'hébergement et de restauration. Pour autant, la diffusion spatiale du phénomène touristique est davantage structurée par la localisation des itinéraires de trekking que par la proximité à Pokhara: le terrain d'étude fait ainsi apparaître un gradient touristique ouest-est (illustration 1), avec deux treks «matures» du côté ouest ( $\mathrm{ABC}$ » et «Balcon des Annapurna » VDC de Dangsing, Dhampus, Lumle et Ghandruk), très fréquentés notamment parce que leur durée relativement brève $\mathrm{e}^{11}$ correspond bien aux attentes des clientèles contemporaines. Mise en place progressivement depuis les années 1980 (Sacareau, 1999), l'armature hôtelière (lodges) et de restauration y est très dense le long des chemins, et certains villages, comme Gorhepani, au pied du célèbre belvédère de Poon Hill, voire Ghandruk (Gurung, 2004), emblématique de la culture Gurung, doivent être regardés comme de véritables stations touristiques. Au centre, sur l'interfluve étroit entre Modi Khola et Mardi Khola qui remonte vers le Machhapucchre, au-dessus de l'étage des villages, le trek du Mardi Himal est en pleine émergence, avec des infrastructures encore limitées mais en voie de densification, et des connexions possibles vers l'est avec le "Machhapuchhre Model Trek» (MMT). Issu d'une expérience volontariste de tourisme communautaire ${ }^{12}$ (Upadhayaya, 2013), avec balisage d'un ensemble de chemins (plusieurs circuits sont envisageables à partir de ce réseau) et soutien à des initiatives locales individuelles et/ ou collectives de logement chez l'habitant (homestays), il enregistre des chiffres de fréquentation nettement plus faibles ${ }^{13}$, et dans lesquels la clientèle nationale fait largement jeu égal avec la clientèle internationale ${ }^{14}$. À la différence des secteurs touristiques plus à l'ouest, où l'essentiel des équipements se concentre à proximité immédiate des itinéraires de trekking, le secteur du MMT présente une mise en tourisme plus diffuse, qui intègre quelques «spots" liés à des activités au développement récent (canyoning, parapente...) ou plus ancien (sources chaudes), et dont l'intensité (nombre et qualité des structures d'hébergement, en particulier) varie en fait surtout en fonction de la vigueur plus ou moins grande des dynamiques locales. D'une manière générale, l'activité touristique apparaît plus développée dans la vallée de la Mardi Khola, au contact du Trek du Mardi Himal, que dans celle de la Seti Nadi, plus à l'est illustration 2). 
Carte 2 - Équipement touristique et centres d'intérêt du périmètre du Machhapuchhre Model Trek

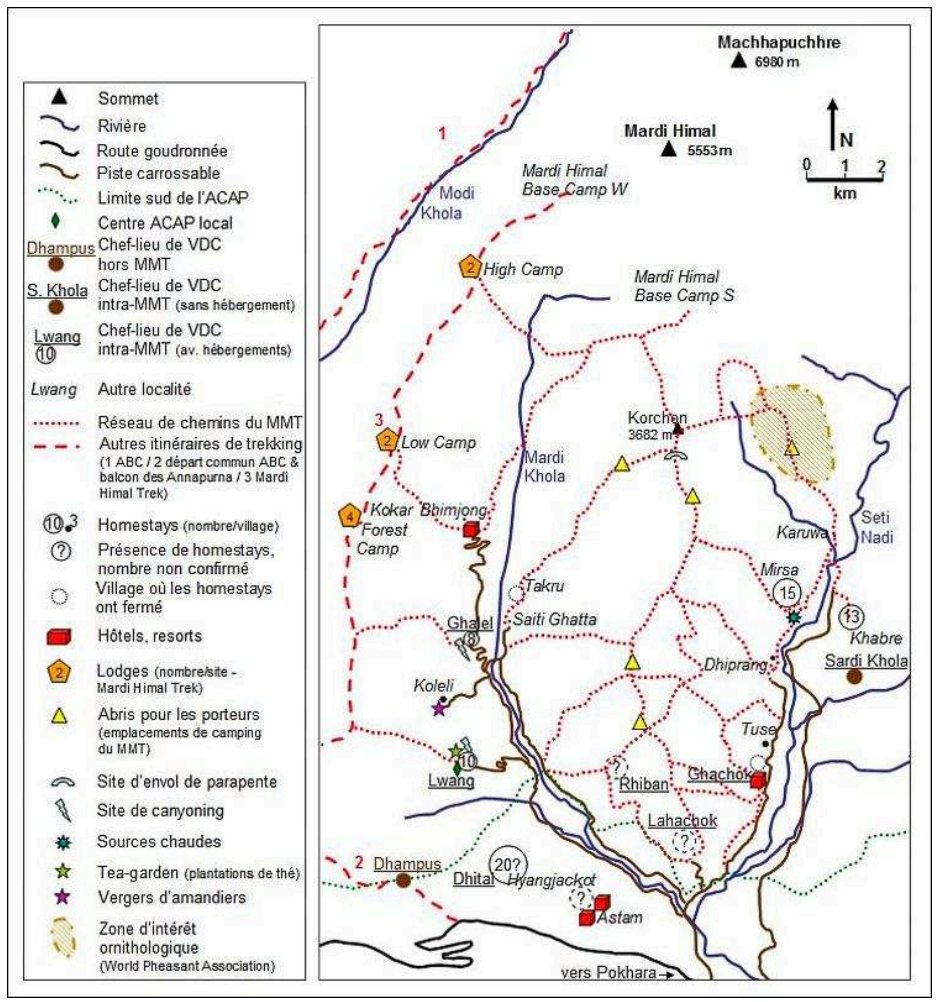

Sources : terrain et entretiens, novembre 2014-avril-mai 2015.

11 Tant dans les formes qu'il prend que dans sa répartition spatiale, ce développement touristique se trouve aussi affecté par le désenclavement rapide des vallées par les nouvelles pistes. Ce dernier entraine en effet souvent la destruction des anciens chemins empierrés empruntés par les trekkeurs, auxquels se superposent des pistes poussiéreuses plutôt rebutantes pour les marcheurs. Lesdites pistes permettent en outre aux touristes d'accéder plus rapidement aux paysages montagnards, en faisant l'impasse sur ceux du piémont: pour certains villages, notamment sur les étapes «aval» du tour des Annapurna, la baisse de fréquentation des hébergements et des commerces par les touristes, induite par la mise en place de la route, est spectaculaire. Mais dans le même temps, la réduction des temps de parcours permet de nouveaux modes de fréquentation, sur des durées beaucoup plus courtes, de l'ordre de la journée ou du week-end: la pratique naissante du canyoning, par exemple, semble s'inscrire dans ce type de logique.

\section{Un puissant courant de migrations de travail}

En dépit des opportunités d'emploi que représente le secteur touristique, cette région, comme de nombreuses autres au Népal, est marquée par le départ vers les emplois urbains ou vers l'étranger d'un grand nombre d'actifs : sans faire partie des districts les plus marqués par cette émigration, le district de Kaski se situe en la matière au-dessus de la moyenne nationale, avec plus de $10 \%$ de population «absente » et un tiers des foyers concernés, chiffres encore supérieurs dans sa partie la plus montagnarde. On compte ainsi $11,1 \%$ de population absente pour les sept VDC du MMT, avec plus de $38 \%$ des foyers qui déclarent que l'un au moins de leurs membres est durablement absent. 
Les chiffres sont à peu près identiques pour les VDC du secteur occidental où l'activité touristique est pourtant plus importante (Dhampus, Ghandruk et Lumle) (illustration 3), et ils correspondent sensiblement à la situation constatée par nos propres enquêtes ${ }^{15}$, qui concernaient majoritairement des ménages impliqués dans des activités touristiques. Le phénomène était encore plus marqué dans l'enquête réalisée sur le village d'Astam (VDC de Dhital) par L.U. Stuhaug (2013, p. 66), dans laquelle plus de la moitié des ménages rencontrés comptait l'un au moins de ses membres travaillant à l'étranger, situation qui avait aussi concerné par le passé l'autre moitié des ménages ${ }^{16}$.

Illustration 3 - Population absente dans les 11 VDC du périmètre d'étude

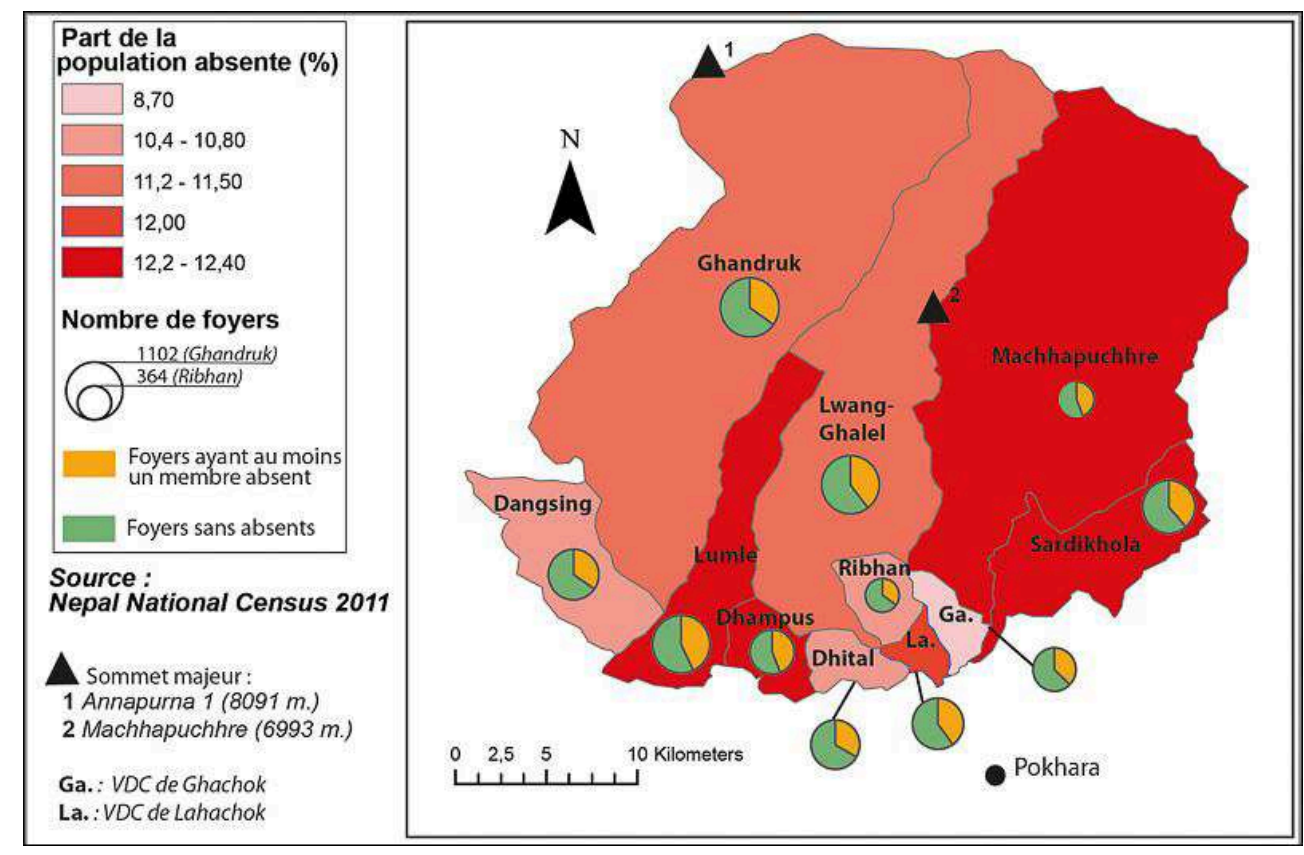

Avec autour d'un dixième de la population absente dans le secteur, l'absence d'au moins un membre concerne entre le tiers (Dhital) et presque la moitié (Machhapuchhre) des foyers.

Conception et réalisation : J. Le Noac'h et P. Derioz, 2016.

Même si cette émigration n'exclut pas des mouvements de plus courte durée et à plus courte distance (notamment vers Pokhara, pour les études et/ou pour prendre un emploi), il s'agit donc avant tout d'une émigration de travail sur plusieurs années vers des pays étrangers. Les destinations les plus citées au cours des entretiens étaient les Pays du Golfe et la Malaisie, avec manifestement des effets de filière de recrutement, en lien avec des agences, qui permettent de partir avec un emploi plus ou moins assuré à l'arrivée, pour des salaires nettement supérieurs à ce qu'ils peuvent espérer gagner au Népal (et $a$ fortiori dans leur village). L'analyse des contrats de travail conduite par T. Bruslé (2012) donnait un salaire mensuel moyen de $183 €$ au Qatar, soit deux à quatre fois plus que la moyenne des salaires en Inde (peu citée dans nos entretiens) ${ }^{17}$, et plus encore par rapport aux salaires népalais. L'expatriation dure plusieurs années, rarement moins de quatre ans, parfois autour d'une dizaine d'années, même si les expatriés ont parfois la possibilité de rentrer au village de façon temporaire, pour une période de vacances - cela n'arrive pas chaque année. En outre, comme le soulignent 0 . Aubriot et T. Bruslé (2012), les jeunes qui émigrent, mis en contact avec d'autres modes de vie (voyage, monde urbain, salaire fixe...), n'aspirent généralement plus à un retour au village pour y vivre de l'agriculture. Si quelques-uns s'installent durablement à 
l'étranger, au point que T. Bruslé (2012) évoque l'existence d'une diaspora népalaise, la plupart d'entre eux souhaite rentrer au pays et employer les économies réalisées pour faire bâtir et habiter en ville (Kathmandu, Pokhara). L'émigration temporaire à l'étranger apparaît ainsi souvent comme une étape dans un processus plus large d'exode rural, qui opère aussi directement, sans passage par l'emploi à l'étranger (Aubriot et Bruslé, 2012).

\section{Un net déséquilibre hommes-femmes}

Cette émigration, qui s'accompagne d'une baisse prononcée du nombre des habitants (-13,1\% entre 2001 et 2011 pour les 11 VDC étudiés), concerne très majoritairement les jeunes hommes. Pour notre terrain d'étude, l'émigration est masculine à $91 \%$ (88 \% pour le Népal dans son ensemble), les hommes durablement partis à l'étranger représentant grosso modo un cinquième de la population masculine totale. Le graphique (illustration 4) suggère indirectement que les hommes absents appartiennent avant tout aux classes d'âge comprises entre 20 et 44 ans, en léger décalage avec le district de Kaski dans son ensemble, pour lequel la classe d'âge 15-34 représentait en $201180 \%$ des absents.

Illustration 4 - Déséquilibre homme-femme par classe d'âge pour les 11 VDC étudiés

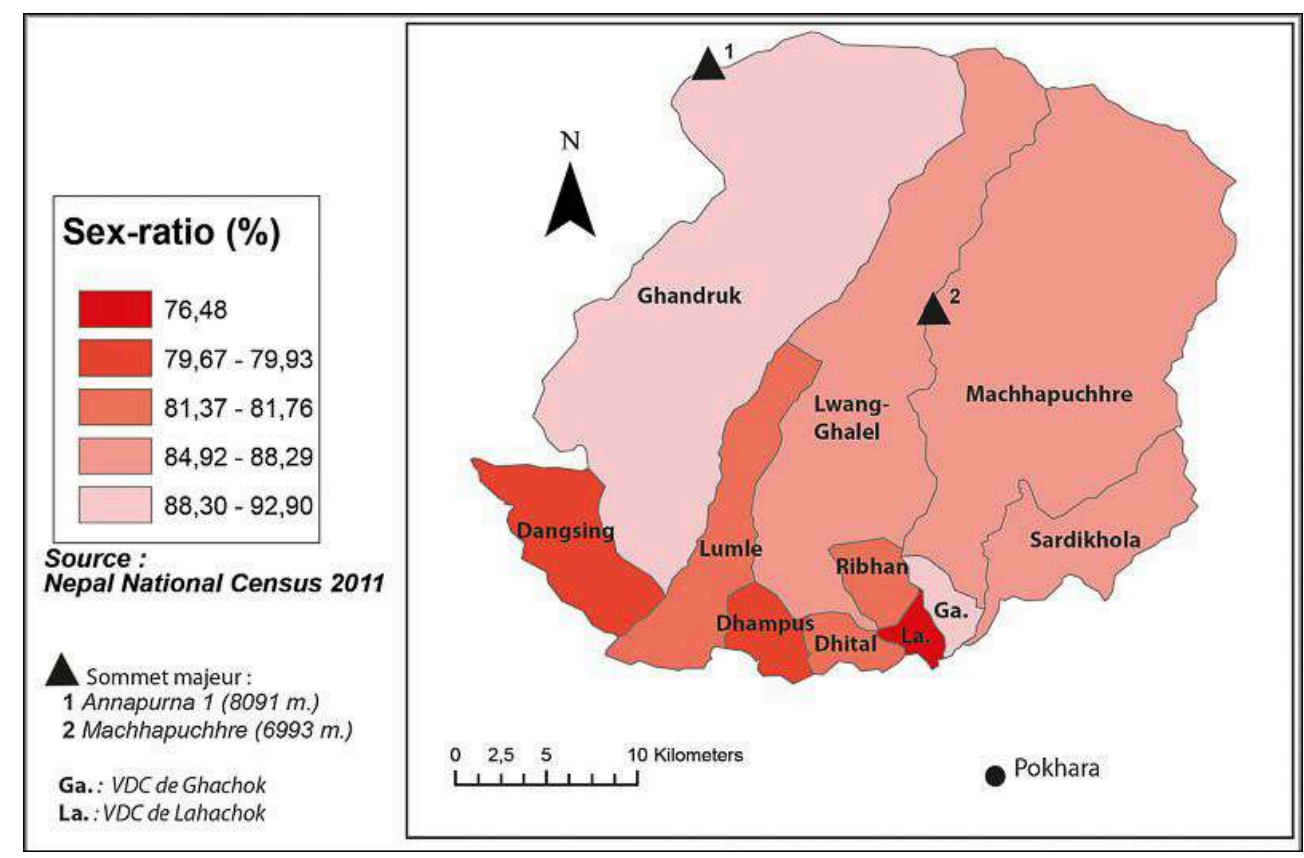

Effectifs par classes d'âge.

Source : Nepal National Census - 2011.

Mais il montre surtout l'ampleur du déséquilibre entre les sexes qui résulte du phénomène migratoire, avec presque un cinquième (19\%) de femmes en plus par rapport à la population masculine présente au sein de notre terrain d'étude, proportion qui est d'une petite moitié de femmes en plus (+48\%) entre 20 et 54 ans, et même de deux tiers $(+66 \%)$ entre 25 et 39 ans. Ce rapport hommes-femmes est certes variable d'un VDC à l'autre - et, au sein de chaque VDC, d'un ward à un autre - (illustrations 5 et 6), moins marqué par exemple à Ghandruk qu'à Dhampus, où il y a pratiquement deux 
fois plus de femmes que d'hommes entre 20 et 39 ans, mais le phénomène est général. Il manque donc beaucoup de bras à l'agriculture villageoise, ce qui se traduit d'abord par des formes de désintensification (pacage sur d'anciennes terrasses de culture) et par l'abandon de terres agricoles au bénéfice des friches puis de jeunes formations forestières. Nos observations rejoignent sur ce point celles réalisées par Khanal et Watanabe (2006) dans le contexte assez similaire du secteur de Sikles, une douzaine de $\mathrm{km}$ plus à l'est. Mais l'absence de nombreux hommes signifie aussi que les femmes se retrouvent souvent de facto à la tête du ménage comme à celle des exploitations ou des entreprises touristiques.

\section{Illustration 5- Déficit en hommes dans la population totale dans les 11 VDC du périmètre d'étude}

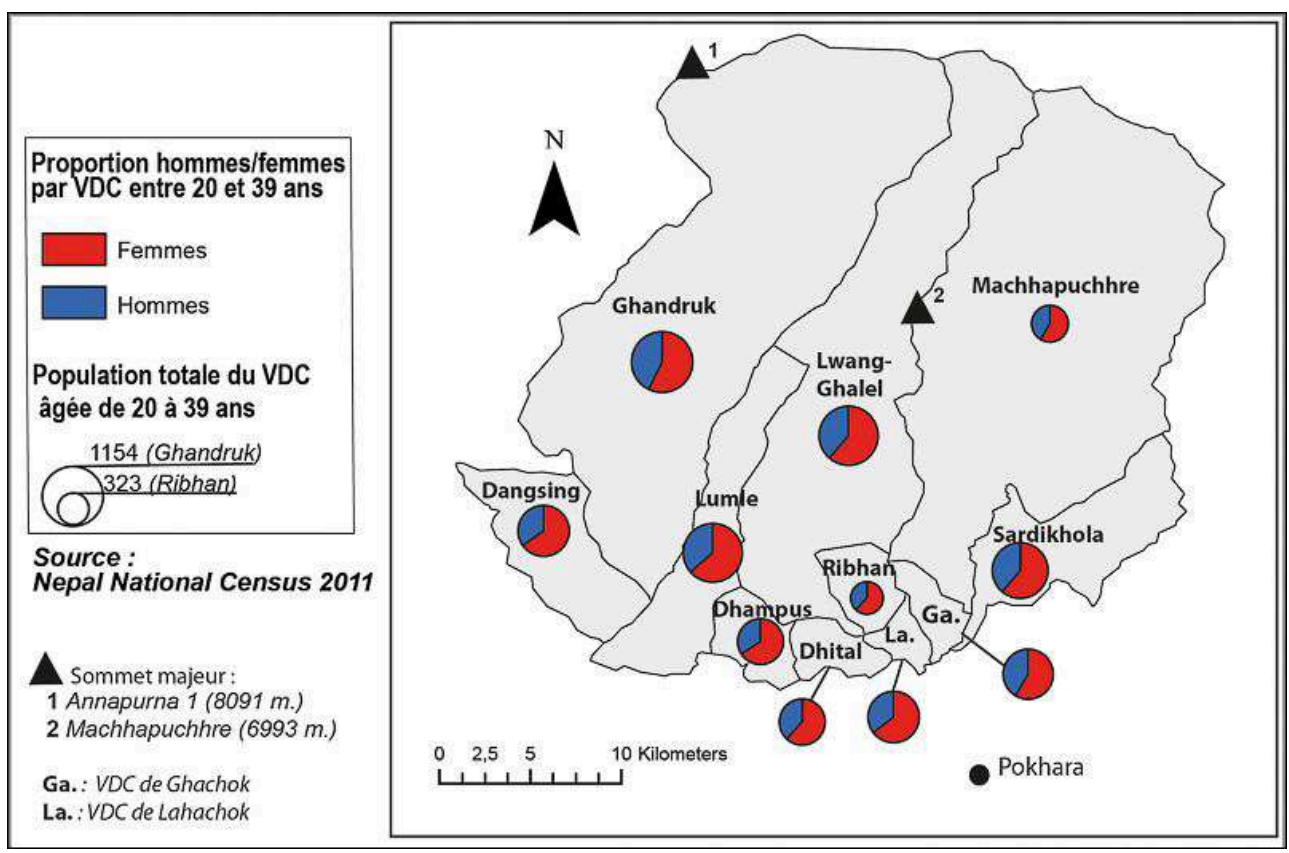

Le déséquilibre du sex-ratio est plus accusé dans les petits VDC du piémont que dans les grands VDC montagnards

Conception et réalisation : J. Le Noac'h et P. Derioz, 2016. 


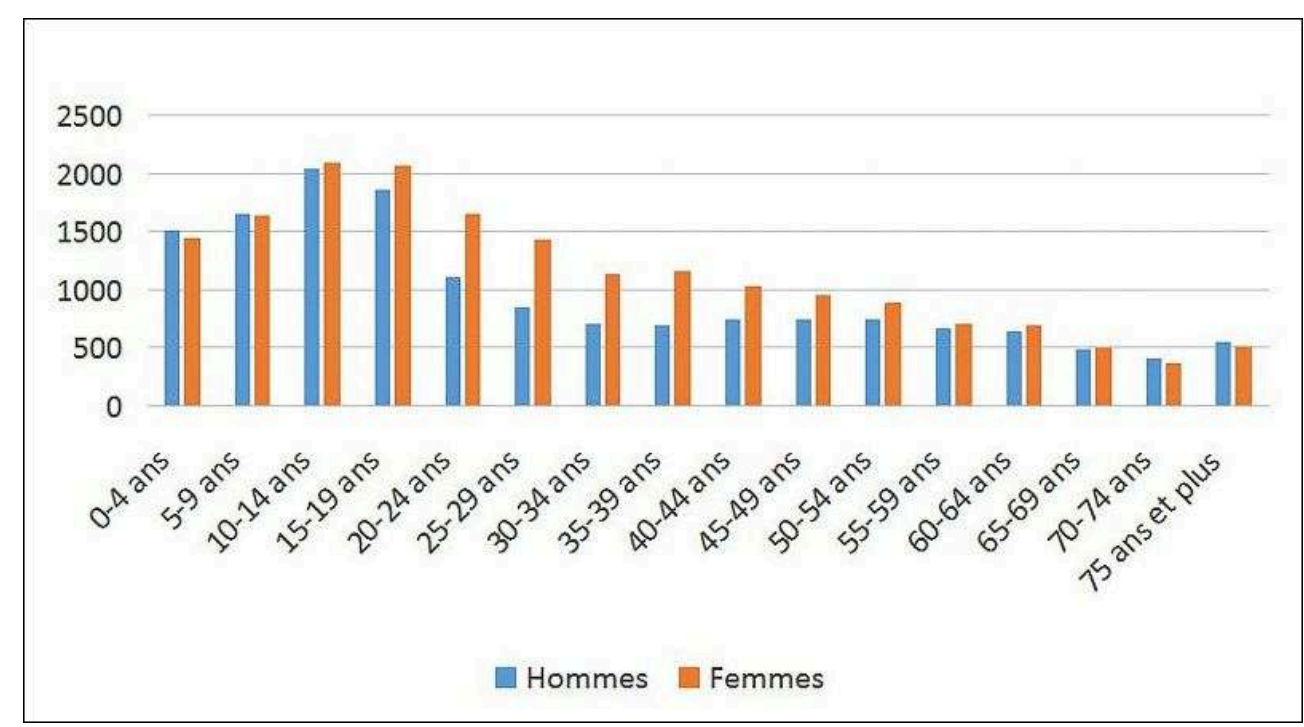

Conception et réalisation : J. Le Noac'h et P. Derioz, 2016.

\section{Absence des hommes et opportunités du développement touristique : une double voie d'émancipation pour les femmes?}

\section{Des réarrangements familiaux temporaires plus ou moins favorables}

Pour l'épouse, le départ du mari qui migre vers l'étranger pour plusieurs années, et rentre seulement de loin en loin pour des vacances de quelques semaines, modifie inévitablement la structure et le fonctionnement du foyer, avec selon les cas des conséquences plutôt favorables ou plutôt défavorables quant à leur autonomisation. Le premier effet de l'absence des hommes, difficile à mesurer dans un contexte général également marqué par un recours croissant aux moyens contraceptifs, par la remontée progressive de l'âge du mariage et par la forte augmentation du nombre des avortements depuis leur légalisation en 2002, semble être la baisse spectaculaire de la fertilité enregistrée depuis 1991 à l'échelle du pays ${ }^{18}$ (Khanal et al., 2013 ; Singh Dangol, 2014 ; Shakya \& Gubhaju, 2016), largement responsable du ralentissement de la croissance démographique $(+1,35 \%$ /an entre 2001 et 2011 , contre $+2,25 \%$ /an au cours de l'intervalle intercensitaire précédent, 1991-2001). Sans que le lien direct avec l'absence éventuelle du mari soit plus facile à établir à l'échelle de notre terrain, aucune des femmes impliquées dans une activité d'accueil touristique que nous avons interrogées n'avait du reste la charge de nombreux enfants. Pour certaines de celles qui étaient en position de gestionnaire d'une structure de type hôtelier (lodge), des arrangements familiaux impliquant la famille élargie (parents, frères et sœurs) organisaient même une prise en charge au moins saisonnière des enfants, favorisant à la fois leur scolarité (en ville) et le travail de leur mère (en montagne).

« Mon mari travaille à Pokhara, et mes deux enfants sont avec lui. Ils vont à l'école

là-bas, le plus grand a 11 ans, ils ne sont jamais montés ici. Je descends de temps en 
temps, mais ici c'est moi qui gère... » (B.G., gérante depuis 2015 d'un lodge vers

$3250 \mathrm{~m}$ d'altitude sur le Mardi Himal Trek, 28/4/16).

\section{réalisés sur la question montrent qu'ils ne favorisent pas automatiquement l'accession} de leurs épouses à un niveau supérieur d'autonomie et de prise de décision (Gartaula, 2007). Cela dépend en particulier de la situation dans laquelle elles se trouvent placées, selon qu'elles restent vivre avec leurs beaux-parents ou qu'elles se retrouvent seules à la tête d'un foyer indépendant (Gartaula et al., 2010, p. 571-573), mais aussi de l'importance des sommes envoyées par l'époux migrant, qui peuvent leur permettre d'échapper au surcroît de travail dans les champs par l'embauche de salariés (Maharjan et al., 2012). En dépit du titre qu'elle a donné à sa monographie du village principalement Gurung de Kalabang ${ }^{19}$ - "I am the Household Head now!" -, H. Kaspar (2005) souligne ainsi la charge de travail supplémentaire que la migration des hommes impose souvent à leurs épouses restées au village, ainsi que la manière dont cette situation tend en définitive à renforcer la division traditionnelle des tâches - activités ménagères et éducation des enfants pour la femme, apport régulier d'argent frais au travers d'une activité salariée à l'étranger pour l'homme. Inexistante pour l'épouse vivant avec sa belle-famille, qui voit généralement sa marge de manœuvre diminuer avec le départ de son mari ${ }^{20}$, l'autonomisation relative de la femme dont le ménage est indépendant de la belle-famille, et qui doit alors gérer seule le foyer et les récoltes, peut en outre se voir remise en cause au retour de l'époux (Gartaula, 2007).

Parmi les études qui s'intéressent à l'impact de la migration du mari sur le fonctionnement du ménage, rares sont celles qui ont concerné des ménages engagés, de manière plus ou moins poussée - spécialisation vs activité complémentaire -, dans des activités en lien avec le tourisme, lesquelles sont souvent regardées comme une voie d'émancipation potentielle pour les femmes népalaises (Sony, 2012; Upadhayaya \& Upreti, 2008; Sherpa, 2007). Là encore, certains des arrangements familiaux induits par ces activités se démarquent nettement de l'alternative entre ménage mononucléaire (le cas échéant dissocié quand le mari est absent) et installation de l'épouse avec ses beaux-parents. Ainsi, à Ghorepani, au pied du site très touristique de Poon Hill, c'est le mari qui est venu vivre chez ses beaux-parents pour y tenir avec sa femme l'hôtel dont ils sont propriétaires. A Landruk, sur l'itinéraire de l'ABC trek, c'est une jeune femme célibataire qui, à l'issue de deux années d'études supérieures de management à Pokhara, est revenue gérer un hôtel de 18 chambres avec l'aide de la jeune épouse de son frère, parti travailler trois ans à Dubaï. Les parents, fondateurs de l'établissement vingt ans plus tôt, sont partis en ville, la laissant en position de chef de l'entreprise familiale.

«J'aime le travail que je fais ici. Mais quand mon frère reviendra, c'est lui qui prendra la gestion de l'hôtel, et je pourrai reprendre mes études. (...) Nos parents habitent maintenant à Pokhara » (A.G., gérante de lodge à Landruk, 19/11/2014).

\section{L'accueil des touristes : un nouveau contexte pour une fonction traditionnelle}

19 Dans leur grande majorité, les tâches qu'implique l'accueil des touristes, de la mise en ordre des chambres à la cuisine en passant par la collecte de bois de feu, le jardinage, le ménage, l'approvisionnement ou la vaisselle, correspondent en première analyse à des tâches ménagères qui relèvent du champ d'intervention traditionnel des femmes. Pour

EchoGéo, 37 | 2016 
Y. Sherpa (2007) il s'agit pour une bonne part d'une «extension du travail domestique » : « Running tourism business was just an extension of household work for Sherpa women ». Dans de nombreux cas, le travail lié à l'accueil s'articule donc très étroitement avec les tâches quotidiennes, qui intègrent aussi le fait de s'occuper des enfants, et amène ainsi les femmes à remplir un «double rôle » (Sony, 2012). La part essentielle qu'elles prennent aux travaux agricoles, notamment en matière de plantation et de récoltes (Gartaula et al., 2010), apparaît ici indissociable de ce double rôle d'hôtesse et de maîtresse de maison. Même lorsque le mari est présent et occupe la place de «manager » de la structure d'accueil, le rôle des femmes, qu'il s'agisse de son épouse ou de salariées, reste déterminant dans son fonctionnement.

20 À l'usage, pourtant, les deux registres de travail - accueil et gestion du ménage - se révèlent assez différents, surtout lorsqu'il s'agit d'accueillir des touristes étrangers dont on ne parle pas la langue, qui s'attendent au respect de certains standards en matière de couchage ou de repas, et expriment des besoins (acheter une bouteille d'eau minérale cachetée au moment d'aller se coucher, par exemple) qu'il n'est pas toujours facile de satisfaire. Outre les menus traditionnels, les lodges qui jalonnent les treks les plus fréquentés se doivent aussi d'offrir à leur clientèle cosmopolite un certain nombre de plats occidentaux (pâtes, pizzas...), répertoriés sur une «carte " que l'action de l'ACAP a tendu à uniformiser. Les attentes sont également différentes en matière de sanitaires, de possibilité de prendre une douche chaude, et aujourd'hui d'accès wifi. Compte tenu du nombre de structures - une vingtaine de lodges et autant de restaurants à Ulleri, une trentaine de lodges et d'hôtels à Ghorepani ${ }^{21} \ldots$-, la concurrence est importante et pousse les structures à tenter de s'aligner les unes sur les autres.

21 En l'absence prolongée du mari, l'épouse restée aux commandes de la structure se retrouve donc confrontée à la fois à des tâches familières mais aussi à beaucoup de nouveautés et d'imprévus, dont plusieurs des personnes interrogées ont confirmé qu'elles s'en étaient souvent trouvées déroutées, voire effrayées. Propriétaire d'un homestay à Lwang, une femme Gurung d'une cinquantaine d'année raconte ainsi combien elle se sentait gauche et embarrassée en présence des touristes, parlant de ses débuts cinq ans plus tôt (2009).

\section{De la situation de fait à la prise de confiance}

Nécessité faisant loi, les femmes restées seules ont pourtant dû faire face, en apprenant peu à peu de leurs expériences successives, dans des registres d'action par ailleurs nettement moins balisés par les normes coutumières ou religieuses que ne le sont, par exemple, les travaux agricoles. Tous les entretiens ou presque font état des difficultés et des interrogations des commencements, associés à une charge de travail accrue par l'absence du conjoint, dont le départ est d'abord vécu comme un "mal nécessaire ", source de contraintes supplémentaires pour celles qui restent. Mais les entretiens témoignent aussi, dans une proportion équivalente, du sentiment partagé d'une prise de confiance progressive, jalonné par l'acquisition de savoir-faire et connaissances nouveaux, et conduisant à des décisions autonomes et à des prises d'initiative, dont ces femmes retirent une légitime fierté.

« Au début, c'était très difficile. Quand les touristes étaient là je ne savais même pas comment me tenir devant eux. Je ne savais pas comment m'y prendre, ni quoi dire.

(...) Ce ne sont pas les formations qui m'ont aidée, j'ai appris toute seule, avec le 
temps, en recevant les gens. Maintenant j'ai tout à fait confiance, je sais leur souhaiter la bienvenue, leur parler, prendre soin d'eux toute seule. Je suis contente de moi. (...) J'aimerais connaître un peu plus d'Anglais... » (P.G., femme Gurung proposant un homestay à Lwang, veuve, dont le fils est à l'étranger, 09/05/2015).

Propriétaire d'un homestay à Lwang, S.G.H. ${ }^{22}$, femme Gurung d'une quarantaine d'années dont le mari est parti travailler au Moyen-Orient, raconte aussi comment elle s'est efforcée d'apprendre un peu d'anglais courant pour échanger avec les touristes, dont elle comprend aujourd'hui suffisamment les demandes pour trouver de l'intérêt personnel à cet échange. Au début, leur désir d'assister ou même de participer à la traite de la bufflonne, à la cuisine ou aux travaux du jardin la surprenait, mais elle dit avoir peu à peu compris en quoi tout cela était nouveau pour eux, et se prêter aujourd'hui avec plaisir à ces échanges, qui lui ont notamment fait réaliser le potentiel que représente le caractère rigoureusement «bio » de leur production de légumes. $\mathrm{Si}$ elle n'exprime pas la même assurance, J.G. ${ }^{23}$, jeune propriétaire de homestay à Ghalel dont le mari se trouve dans un pays du Golfe, gère toute seule l'accueil et l'approvisionnement de sa petite structure qui reçoit surtout des clients népalais, et manifeste justement son désir de formation au métier de l'accueil, comme sa volonté d'élargir la gamme de ses compétences culinaires. Quant à L.G., gérante d'un homestay à Siddhing, l'absence de son mari est seulement liée au fait qu'il tient un lodge sur l'itinéraire du Mardi Himal Trek, à 4 heures de marche de là ; c'est elle qui se rend une fois par mois à Pokhara pour acheter l'approvisionnement des deux établissements, qui fonctionnent en complémentarité et se renvoient les clients.

«J'aime beaucoup recevoir des touristes, et parler avec eux. (...) C'est en parlant avec eux que j'ai vu qu'il fallait installer la douche avec l'eau chaude. Depuis qu'il y a cette douche, j'ai commencé à compter les touristes qui viennent, parce qu'il me faut prévoir pour le gaz » $(23 / 11 / 14)$.

Les mots des femmes rencontrées sont souvent les mêmes, et renvoient aux mêmes points clés du développement de l'autonomie et de la confiance :

- l'aptitude à assumer le contact avec les clients, qui passe par une certaine maîtrise de l'anglais quand il s'agit de clientèle internationale ;

- l'apprentissage de la gestion fine de l'approvisionnement et des fournitures dans un contexte d'incertitude, la fréquentation touristique, par définition irrégulière et aléatoire, imposant une organisation différente de celle qui prévaut pour le foyer ;

- la capacité à fonder sur l'analyse des demandes des clients la mise en place de prestations nouvelles (vente de boissons, artisanat textile, tapis...) et des projets d'amélioration (sanitaires, meilleure literie, extensions...), qui mobilisent parfois directement l'argent envoyé de l'étranger par les époux ou les frères ;

- l'insertion active dans un réseau collectif de partenaires économiques, fournisseurs, clients (agences urbaines de Pokhara) ou partenaires locaux exerçant la même activité, enfin. Ce dernier aspect apparait particulièrement déterminant dans le système des homestays, là où la mise en place plus ancienne des lodges le long des principaux axes de trekking relève davantage des règles de l'entreprise individuelle (Sacareau, 1999).

\section{Rôle des institutions et des démarches collectives en matière d'empowerment des femmes}

L'« autonomisation des femmes" (women's empowerment) fait au Népal très souvent partie des objectifs explicites des institutions et des ONG, nationales, régionales ou 
locales, particulièrement en matière de développement par le tourisme (Acharya, 2013 ; Upadhayaya, \& Upreti, 2008). Les travaux de D. Gurung (1995) puis M. Gurung (2004), qui analysent avec précision les modalités de l'action conduite par l'ACAP au sein des VDC du périmètre qu'elle administre ${ }^{24}$, attestent de l'effet levier des politiques conduites en la matière, même si les rapports sont parfois compliqués entre l'ACAP et les différentes communautés locales. Au début des années 1990, l'aide à la mise en place et la stimulation par l'ACAP des «Groupes des Mères » (Ama Toli) locaux - le premier a été créé à Ghandruk en 1992 -, dans le cadre de son Women's Development Program, a par exemple permis la mise en place de cours d'alphabétisation pour les adultes, de bourses d'études pour les jeunes filles, l'ouverture d'une crèche à Ghandruk, et le lancement du programme DWET (Developing Women's Entrepreneurship in Tourism). Après des débuts au ralenti, ce programme, comportant des sessions de formation, un accès au crédit pour la création d'activités et un suivi des projets a permis à plusieurs dizaines de femmes de bénéficier de ces formations et quelques-unes ont franchi le pas de la création d'activités complémentaires liées au tourisme, le plus souvent de petits ateliers (microélevages, couture...) ou de petits commerces. Les réalisations peuvent paraître modestes, mais le cadre collectif dans lequel elles se sont déployées, joint à l'effet d'émulation engendré par le succès de quelques pionnières, comme la première femme de Ghandruk à avoir ouvert un lodge devenu aujourd'hui l'un des plus réputés ${ }^{25}$, constitue l'un des vecteurs majeurs de la montée en puissance du rôle des femmes dans le tourisme.

Ce sont sensiblement les mêmes ressorts - appuis institutionnels, volonté d'inscrire l'action dans des approches participatives et dynamique collective animée par les Groupes des Mères initiés par l'ACAP - que l'on retrouve dans l'expérience du Machhapuchhre Model Trek, déjà évoquée plus haut (Upadhayaya, 2011, p. 27-30; Thapaliya, 2012). Là encore, les femmes - que leur mari soit présent ou pas - ont constitué le public cible des formations et de l'aide à la création d'activités qui a débouché sur l'ouverture de plus d'une cinquantaine de homestays (carte de l'illustration 2 et photos des illustrations 7 et 8), et leur logique d'exploitation est fondamentalement collective : elle repose sur de multiples échanges de produits et de fournitures, sur l'entraide informelle au sein de groupes de homestays voisins, sur la gestion collective de l'accueil de groupes importants, sur la mise au point de petits spectacles folkloriques pour les touristes, et surtout sur la répartition égalitaire des clients entre les opérateurs, sous l'autorité du Groupe des Mères. Dans le domaine du tourisme comme dans les autres domaines d'intervention du Groupe des Mères (éducation des enfants, santé, propreté du village, lieux de culte...), c'est largement son caractère institutionnel qui fonde la crédibilité du groupe, autorise éventuellement l'emprunt, force un respect que viennent renforcer les réalisations. La dynamique collective, qui favorise l'entraide et l'échange d'expérience, exerce ainsi en retour un effet bénéfique sur le statut individuel des femmes, ce qu'elles expriment parfois au détour des entretiens d'une manière assez paradoxale, non pas comme une conquête mais plutôt comme la révélation de capacités auxquelles elles n'imaginaient pas avoir accès. D'une manière très générale, en effet, elles interrogent peu leur position sociale et ne la vivent pas comme discriminante (Kaspar, 2005, p. 113-115), dans un contexte montagnard où le rapport hommes-femmes a par ailleurs souvent été décrit comme moins déséquilibré et inégalitaire que pour le reste du Népal, en particulier au sein des groupes tibéto-birmans (Sherpa, 2007 ; Pignède, 1993). 
Illustration 7 - Les homestays de Lwang, panneau d'acceuil avec leur localisation

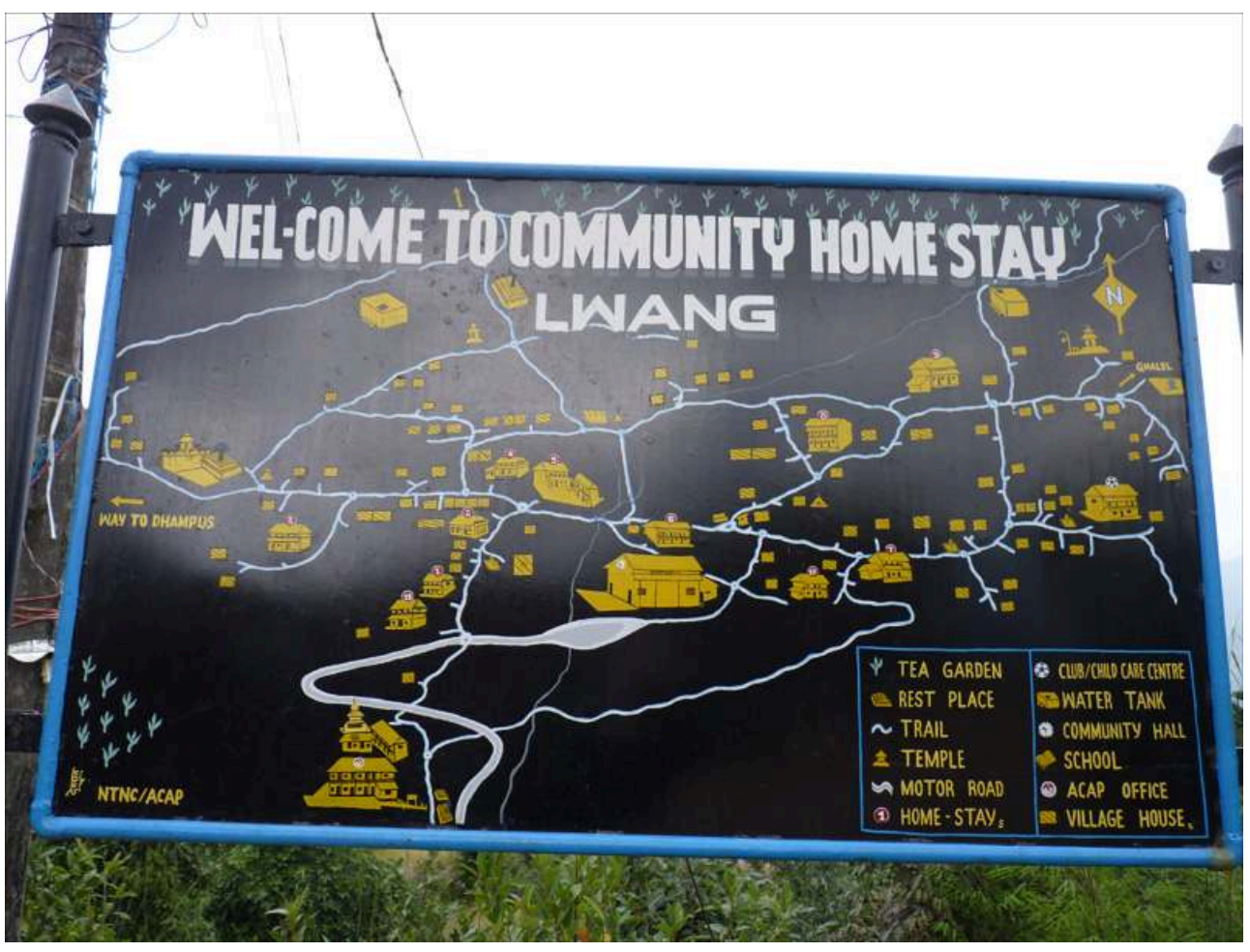

Auteur: P. Derioz, novembre 2014. 


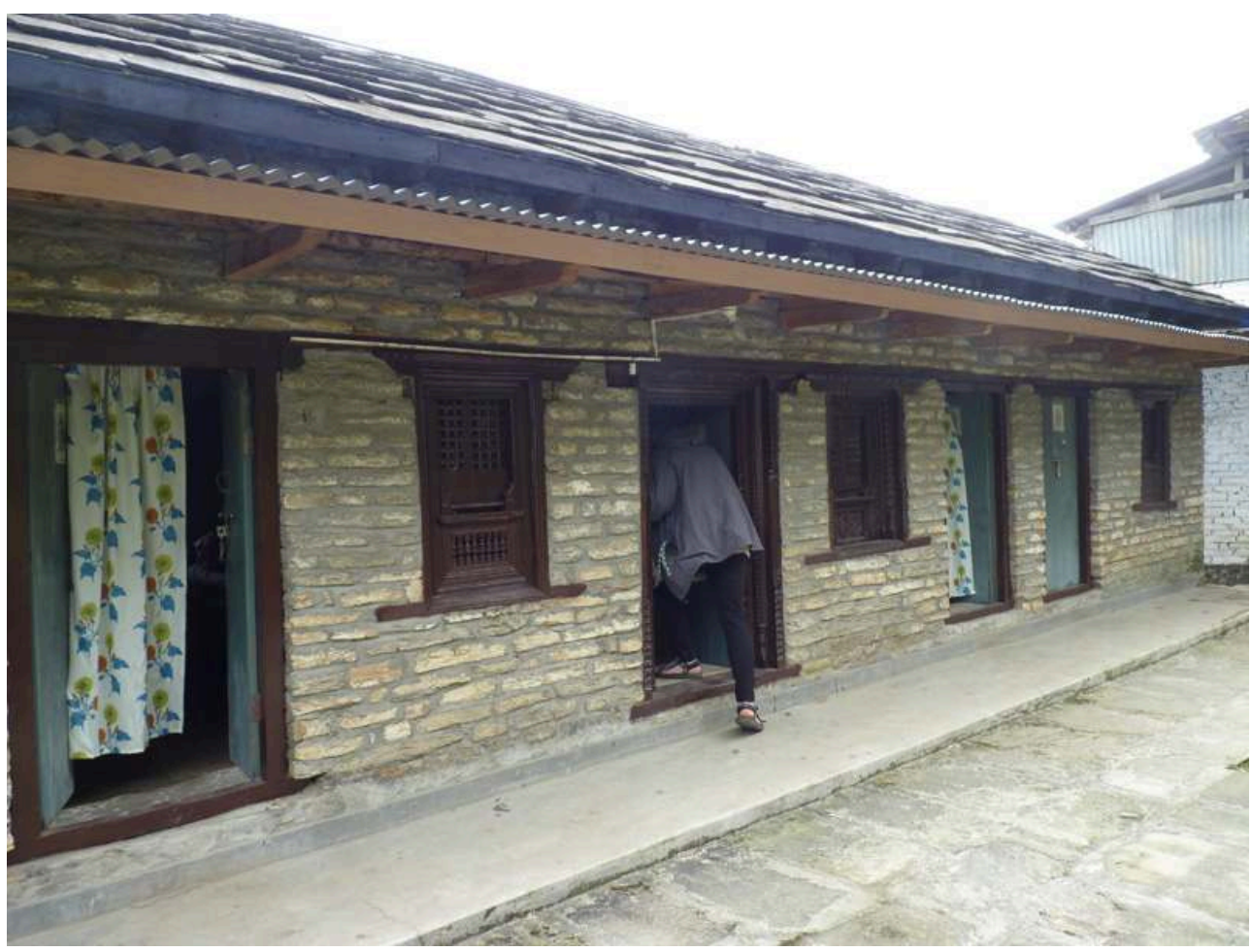

Géré par une femme et sa belle-fille, les trois fils de la première, dont le mari de la seconde, travaillent à l'étranger. Les quatre chambres de ce grand homestay sont dans un bâtiment séparé de leur habitation.

Auteur : P. Derioz, novembre 2014.

\section{Disparité des situations individuelles et collectives, entre autonomisation, tensions et formes d'exclusion}

\section{Les métiers du tourisme autres que ceux de l'hébergement : manque de reconnaissance et discriminations persistantes pour les femmes}

Si les avancées constatées sur le terrain sont bien réelles, la moindre reconnaissance (en particulier salariale) que la société népalaise accorde au travail féminin (Thapa Adhikari, 2013, p. 85) n'épargne pas pour autant le secteur touristique. À l'échelle du pays, les données existantes sur l'emploi féminin dans ce secteur montrent d'ailleurs que si les femmes ne sont sans doute pas loin d'en représenter la moitié, les emplois qu'elles occupent sont majoritairement les moins qualifiés (Sherpa, 2007). Par ailleurs, alors que les formes d'empowerment qui découlent pour les femmes de la gestion plus ou moins autonome d'hébergements touristiques semblent plutôt bien acceptées au sein de la société locale, y compris par les hommes, il n'en va pas toujours de même pour l'ensemble des métiers du tourisme. Autant il n'est pas rare de voir les femmes à la tête de lodges ou de homestays encouragées et complimentées par leurs maris ou par les guides qui fréquentent leurs établissements pour les savoir-faire et les compétences qu'elles ont acquis, autant elles se heurtent à des réticences plus fortes dès lors qu'elles sortent de ce cadre, qui relève malgré tout encore de la sphère domestique, et briguent à l'extérieur un emploi salarié. Parce que la conception traditionnelle du rôle de la 
femme la renvoie avant tout aux tâches domestiques et à la garde des enfants, une condamnation sociale plus ou moins vive entoure encore souvent l'exercice des métiers de porteur ou de guide au féminin (Sony, 2012). Sur les 116 agences de trekking enregistrées au sein de la TAAN à Pokhara, du reste, 4 seulement sont dirigées par des femmes. Le positionnement et le discours de ces pionnières se font ici plus nettement militants, et témoignent de leur sentiment de devoir conquérir reconnaissance et indépendance économique.

«Maintenant les femmes sont de plus en plus acceptées dans le milieu du tourisme, même si certains hommes sont très fermés d'esprit : ils estiment que ce n'est pas un travail pour les femmes car c'est difficile physiquement [porteur et/ou guide]. Ils pensent qu'elles ne peuvent pas être de bonnes guides, et surtout que leur place n'est pas dans un emploi autre que celui du foyer. (...) Le tourisme est un moyen de donner un salaire aux femmes : même si elles travaillent énormément au foyer et dans les champs, ce travail n'est pas récompensé par un salaire. En étant guide ou en gérant un hôtel, ce travail est reconnu, les femmes peuvent être indépendantes. Enfin, plus ou moins » (Y.B., femme guide au sein de la première agence de trekking féminine de Pokhara, créée en 1998, Pokhara,22/05/2016).

«Ce que j'ai le plus envie de montrer aux touristes, ce sont des zones vierges et sauvages, et le dur travail des femmes dans ces zones: on dit toujours qu'ici les femmes n'ont pas de travail, car oui elles n'ont pas d'emplois et pas de salaires, mais elles ont le plus dur des travail, dans les champs, en plus du rôle de femme au foyer » (D.L.R., femme guide, , Hile, Trek du Balcon des Annapurna,09/04/2016).

\section{Un développement touristique qui conforte les inégalités des sociétés locales}

Les opportunités de travail et de revenus que ménage le développement touristique là où il se manifeste, par ailleurs, excèdent largement le seul champ des métiers et activités directement liées au tourisme: les multiples besoins des touristes et l'approvisionnement des divers types d'hébergement fournissent aussi à de nombreuses familles des occasions de vendre des produits agricoles ou de fournir des services (portage, construction, ménage...). Mais, sans être pour autant toujours négligeables, les profits qu'elles en retirent sont généralement limités. La gestion d'un homestay, et a fortiori la possession d'un lodge, représentent des revenus plus conséquents, mais ils impliquent d'emblée la possession par les ménages concernés d'un certain nombre d'atouts en termes de patrimoine (grande maison, foncier...), de capacité d'investissement (économies, montant élevé des versements envoyés par les hommes travaillant à l'étranger) et d'aptitudes intellectuelles à la gestion (éducation scolaire). Essentiels lors de l'investissement initial, les moyens financiers sont aussi indispensables pour suivre le rythme de modernisation des structures imposé par la concurrence. Au sein de sociétés rurales dans lesquelles les inégalités de caste et les inégalités socio-économiques se renforcent mutuellement, ce sont donc sans surprise les familles les plus riches, membres des groupes ethniques (Gurungs) et des castes (Brahmane) dominants ${ }^{26}$, qui se retrouvent à la tête des hébergements comme d'ailleurs des agences de trekking. S.K. Nepal (2002, p. 42) souligne ainsi la manière dont l'activité touristique a souvent eu tendance à creuser les inégalités au sein des communautés villageoises.

Les formes d'empowerment qu'entrainent pour les femmes ces nouvelles fonctions liées à la gestion d'hébergements touristiques apparaissent donc aussi largement réservées aux femmes qui appartiennent aux catégories sociales dominantes, à l'image 
de ce que M. Oiry-Varacca $(2014 ;$ 2016) observe au Maroc dans le Haut-Atlas occidental. Inscrite dans une approche intersectionnelle ${ }^{27}$ (Crenshaw, 1989 ; Valérie, 2007 ; Shields, 2008), son analyse montre de même que «le tourisme ne modifie pas radicalement les relations de genre normées socialement", dans la mesure où il ne fait évoluer les relations de genre que " graduellement, en partant du haut et non de la base : il change la donne pour les femmes des élites, ce qui reflète et renforce les rapports de pouvoir locaux ». En versant sud des Annapurna, les appartenances ethniques et de caste demeurent en dernière analyse un facteur clé: socialement et économiquement discriminés, les foyers appartenant aux basses castes et au groupe des intouchables restent très largement à l'écart du développement touristique, faute de moyens, de formation et de considération de la part du reste de la société (Sherpa, 2007 ; Thapaliya, 2012). Les ménages Dalits ne prennent pratiquement aucune part à l'offre touristique de Lwang (Dawadi \& Upadhayaya, 2013, p. 279), où leurs membres féminins siègent au sein d'un Groupe des Mères soigneusement distinct de celui des femmes Gurung ou Brahmanes, et globalement peu actif. Tout au plus se voient-ils impliqués dans les spectacles «traditionnels » qui sont systématiquement proposés aux touristes, et engendrent occasionnellement des recettes aléatoires, dans lesquels les acteurs locaux dominants aiment à voir une forme de partage des fruits du développement touristique.

«Les maisons des Dalits ne sont pas convenables pour recevoir des touristes, elles sont trop petites. Ils n'ont pas d'instruction pour ça. (...) Mais avec les spectacles qui sont organisés pour les visiteurs, les chants et la musique, et les danses, il reçoivent un peu d'argent. Comme ça le tourisme profite à tout le monde dans le village " (D.K.G., présidente gurung du Groupe des Mères de Lwang, 20/11/2014).

30 Mais lorsque ni les revenus du ménage, principalement liés au travail agricole salarié, ni les envois d'argent de l'époux parti à l'étranger (Thieme et al., 2011, p. 67) ne suffisent, le secteur du tourisme n'offre guère à ces femmes que des emplois temporaires peu qualifiés, de ménage ou de jardinage dans les lodges les plus importants, ou encore de portage (Sony, 2012). Le surcroît de charge de travail qui en résulte ne s'accompagne guère dans de tels cas d'une prise d'autonomie ou de perspectives d'ascension sociale - même si de rares cas montrent qu'elle est toujours possible.

\section{Le jeu complexe des inégalités spatiales}

31 La position des villages par rapport aux principaux itinéraires de trekking et aux attractions touristiques majeures, enfin, détermine aussi en bonne partie la gamme des possibles. Les flux touristiques très différents entre la vallée de la Modi Khola (52 000 touristes étrangers sont passées en 2013 par Gandhruk sur l'ABC trek, auxquels s'ajoutent quelques 80000 touristes népalais souvent non trekkeurs ${ }^{28}$ ), celle de la Mardi Khola (682 touristes à Lwang la même année, dont $75 \%$ de népalais) et celle de la Seti Nadi (quelques centaines de touristes par an, plutôt des nationaux) n'offrent à l'évidence pas le même niveau d'opportunités aux communautés villageoises. De même le développement touristique limité du village d'Astam étudié par L. Stuhaug (2013, p. 62) s'explique-t-il avant tout par sa situation à l'écart.

Mais là encore, le jeu complexe des liens d'appartenance à des réseaux ethniques, familiaux ou professionnels, qui jouent à différentes échelles, reste important (Létang et al., à paraître). Le fait d'être intégré dans le réseau des fournisseurs habituels des 
agences de Pokhara, pourvoyeuses de clientèle - à l'occasion en échange de commissions -, joue par exemple un rôle certain dans le remplissage des structures touristiques par la venue de groupes accompagnés. De même l'investissement de la TAAN dans l'expérience du Machhapuchhre Model Trek à la fin des années 2000, dans deux vallées jusque-là peu ou pas concernées par les activités touristiques correspondil à la période où son président était originaire de l'un de leurs villages, où il a d'ailleurs lui-même développé un petit resort touristique en 2011 en association avec un partenaire français qui a apporté la plus grande part des capitaux. Quant à la géographie des villages où s'est engagée depuis une activité de homestays (Lwang, Khabre, Mirsa, cf. illustration 2), elle n'est guère éloignée de celle des villages à dominante Gurung, même si entre eux se manifeste davantage un esprit de concurrence qu'une volonté de collaboration. Aucun homestay n'a en revanche été ouvert dans le village Magar de Bharabari, pourtant nettement mieux placé que ses voisins Khabre et Mirsa par rapport aux sources chaudes de Kharpani, principal attracteur touristique du secteur.

\section{Conclusion}

L'image renvoyée par le terrain n'est donc pas univoque. Elle montre de nombreux exemples de réussites individuelles ou collectives, liées au développement touristique, dans lesquelles les femmes qui étaient en position - sociale, familiale, économique, intellectuelle... - d'en profiter ont gagné en autonomie et en indépendance. Sans doute l'appel d'air engendré par l'émigration de très nombreux hommes a-t-il contribué à ouvrir pour elles le champ des possibles, mais force est de constater que l'on rencontre aussi en versant sud des Annapurna des situations où le rôle de la femme dans l'entreprise touristique reste important, voire prépondérant, alors que le mari est toujours présent. Hors du milieu des guides, dans lequel les femmes restent peu nombreuses et ont toujours des difficultés à faire accepter leur statut, les formes d'empowerment qui découlent de la gestion ou de la co-gestion de structures d'hébergement ne s'opèrent d'ailleurs pas dans un climat marqué par un antagonisme hommes-femmes, mais bien davantage par le biais d'arrangements et de compromis "pragmatiques » au sein du foyer, qui modifient le statut de la femme à l'échelle de la famille par l'acquisition de compétences, l'exercice de responsabilités et l'obtention de revenus. Ces évolutions, telles qu'elles sont perçues à l'échelle des communautés villageoises ou décrites par les responsables d'agence masculins de Pokhara, semblent bien davantage saluées que critiquées.

Dans le même temps, l'existence d'autres formes de discrimination que celles liées au genre, qui généralement se cumulent, tend à réserver les possibilités d'empowerment aux femmes issues des groupes sociaux dominants. Sauf exception, elles ne sont pas accessibles aux népalaises de basse caste, même si plusieurs témoignages s'accordent sur la lente évolution des mentalités et des pratiques enclenchées au moment de la guerre civile, sous la pression des maoïstes.

«Les Dalits étaient nombreux à s'être engagés du côté des maoïstes pour que les choses changent. Elles bougent doucement. C'est devenu possible pour moi d'en employer ici [i.e. dans son lodge], et le tourisme joue aussi un rôle positif. (...) Mais ils n'ont toujours pas vraiment le droit d'entrer dans la maison de mes parents. C'est important de garder la tradition, pour le tourisme aussi. Mais ça, ça n'est pas 
de la tradition, c'est de la discrimination pure et simple » (B.R.D., 56 ans, Brahmane, propriétaire de lodge dans la zone du Machhapuchhre Model Trek, 24/11/2014). d'une nouvelle Constitution en net repli dans ce domaine sur plusieurs points essentiels (transmission de la nationalité, héritage, droit à l'avortement...), et qui a suscité de vives protestations de la part des mouvements féministes, reste ainsi indissociable des autres formes de discriminations, à commencer par celles qui découlent du système des castes. Mais il n'en reste pas moins que les exemples plutôt urbains des accomplissements professionnels de femmes guides ou directrices d'agence, de boutiques, de restaurant d'hôtels (Acharya, 2013, p. 160), comme la part d'autonomie acquise par certaines dans le contexte rural, montagnard et multiethnique de notre territoire d'étude, ne peuvent que contribuer positivement à l'évolution de l'image et du statut de la femme népalaise.

\section{BIBLIOGRAPHIE}

Acharya S., 2013. Women's participation in tourism in Pokhara. In B.R. Upreti, P.K. Upadhayaya \& T. Sapkota edts, Tourism in Pokhara. Issues, Trends and Future Prospects for Peace and Prosperity, chap.

7, Pokhara Tourism Coucil, NCCR North-South, Nepal Center for Contemporary Research, Kathmandu, p. 155-172.

Acharya D., Bell J., Simkhada P., van Teijlingen E., Regmi P., 2010. Women's autonomy in household decision-making: a demographic study in Nepal. Reproductive Health Journal, vol. 15, $\mathrm{n}^{\circ}$ 7. https://reproductive-health-journal.biomedcentral.com/articles/10.1186/1742-4755-7-15

Acharya M. (coord.), 2007. Gender Equality and Empowerment of Women in Nepal. UNFA Report (United Nations Population Fund), 94 p. http://un.org.np/node/10577

Acharya M., 2003. Changing Gender Status: Achievements and Challenges; Population Monograph of Nepal, vol. II, chap. 18, CBS, National Planning Commission, Kathmandu, Nepal, p. 217-150.

Ashley C., Boyed C., Goodwin H., 2000. pro - poor tourism: Putting Poverty at the Heart of the Tourism Agend", ODI, Natural Resources Perspectives, n 51. https://www.odi.org/sites/odi.org.uk/ files/odi-assets/publications-opinion-files/2861.pdf

Ateljevic I. (dir), 2008. Women empowerment through tourism. Wagenigum University, 136 p. http:// cf.cdn.unwto.org/sites/all/files/pdf/report-

women_empowerment_through_tourism_by_irena_ateljevic_2008.pdf

Aubriot O., Bruslé T., 2012. Agriculture paysanne népalaise et phénomènes migratoires : de la complémentarité à la rupture des liens ? Presses de Sciences Po | Autrepart, nº 62 (2012-3), p. 141-158. http://www.cairn.info/revue-autrepart-2012-3-page-141.htm

Bennett L., 2005. Gender, Caste and Ethnic Exclusion in Nepal: Following the Policy process from Analysis to Action. Arusha Conference, "New Frontiers of Social Policy", 12-15 ${ }^{\text {th }}$ December 2005. http://siteresources.worldbank.org/INTRANETSOCIALDEVELOPMENT/Resources/ Bennett.rev.pdf 
Bhattarai A.K., 2014. How Rural Women Experience Disempowered in Nepal? Master in Social WorkComparative Perspective, University of Nordland, Norway, 96 p. https://brage.bibsys.no/xmlui/ bitstream/handle/11250/220969/Bhattarai.pdf?sequence=1

Borghi R., 2003. L'évolution du rôle de la femme marocaine sur la place Jama' al Fna de Marrakech. In Mouline S. (dir.), Jama' al Fna, entre art et bazar. Rabat, Direction de l'Architecture, p. 52-62.

Brink J.H., 1991. The Effect of Emigration of Husbands on the Status of Their Wives: An Egyptian Case. International Journal of Middle East Studies, vol. 23, n 2, p. 201-211.

Bruslé T., 2012. What's New in The Gulf? New Technologies, Consumption and Display of Modernity Among Nepalese Workers in Qatar. e-migrinter, $n^{\circ}$ 8, p. 59-73.

Calvès A., 2014. L'empowerment des femmes dans les politiques de développement : Histoire d'une institutionnalisation controversée. Regards croisés sur l'économie, $\mathrm{n}^{\circ} 15$ (2/2014), A. Benhenda \& F. Salin (edts) : « Peut-on faire l'économie du genre? », ed. La Découverte, p. 306-321.

Calvès A., 2009. "Empowerment" : généalogie d'un concept clé du discours contemporain sur le développement. Revue Tiers Monde $\mathrm{n}^{\circ} 200$ (4/2009), p. 735-749. www.cairn.info/revue-tiersmonde-2009-4-page-735.htm

Campus D., 2016. Does Land Titling Promote Women's Empowerment? Evidence from Nepal. Working Paper $n^{\circ}$ 3, DISEI, Università degli Studi di Firenze, 31 p. http://www.disei.unifi.it/upload/ sub/pubblicazioni/repec/pdf/wp03_2016.pdf

Dawadi B.R., Upadhayaya P.K., 2013. Role of tourism in urban-rural integration: Reflexions from trekking (adventure) tourisme in Pokhara. In B.R. Upreti, P.K. Upadhayaya \& T. Sapkota edts, Tourism in Pokhara. Issues, Trends and Future Prospects for Peace and Prosperity, chap. 11, Pokhara Tourism Coucil, NCCR North-South, Nepal Center for Contemporary Research, Kathmandu, p. 251-287.

De Haas H., Van Rooij A., 2010. Migration as Emancipation? The Impact of Internal and International Migration on the Position of Women Left Behind in Rural Morocco. Oxford Development Studies, vol. 38, n 1, p. 43-62. http://www.tandfonline.com/doi/abs/ $10.1080 / 13600810903551603$

Department of Foreign Employment, 2014. Labour Migration for Employment. A Status Report for Nepal: 2013-2014, Government of Nepal, Ministry of Labour and Employment, 60 p. https:// asiafoundation.org/resources/pdfs/MigrationReportbyGovernmentofNepal.pdf

Ferguson L., 2011. Promoting gender equality and empowering women? Tourism and the third Millennium Development Goal. Current Issues in Tourism, vol. 14, n 3, p. 235-249.

Gartaula H.-N., Niehof A., Visser L., 2010. Feminisation of Agriculture as an Effect of Male Outmigration: Unexpected Outcomes from Jhapa District, Eastern Nepal. The International Journal of Interdisciplinary Social Sciences, Vol. 5, nº 2, p. 565-577. https://www.researchgate.net/ publication/46383452_Feminisation_of_agriculture_as_an_effect_of_male_outmigration_Unexpected_outcomes_from_Jhapa_district_eastern_Nepal

Gartaula H.-N., 2007. Gendered Impact of Male Out-migration in Rural Households: A short review of literature. The Thesis Abstract online, Sociology of Consumers and Households, Department of Social Sciences, Wageningen University, The Netherlands http://www.thesisabstracts.com/ ThesisAbstract_323_Gendered-Impact-of-Male-Out-migration-in-Rural-Households-A-shortreview-of-literature.html 
Giri K., 2012. Gender reforms in Nepal: Retreat or Predicaments? A review of the politics and practice of women's rights. Southasia Institute of Advanced Studies/Alliance for Social Dialogue, Kathmandu, Nepal, 51 p. http://asd.org.np/wp-content/uploads/2015/03/gender_reforms_in_nepal.pdf

Gurung Y.-B., 2012. Migration From Rural Nepal: A Social Exclusion Framework. Himalaya, the Journal of the Association for Nepal and Himalayan Studies, vol. 31: $n^{\circ} 1$, article 12. http:// digitalcommons.macalester.edu/himalaya/vol31/iss1/12

Gurung M., 2004. Women and Development in the Third World. A Case Study from Ghandruk, Nepal. WWF Nepal Program Office, $104 \mathrm{p}$.

Gurung D., 1995. Tourism and Gender: Impact and Implications of Tourism on Nepalese Women. ICIMOD, Discussion paper, coll. Mountain Enterprises and Infrastructure, Kathmandu, 84 p. http:// lib.icimod.org/record/24936/files/attachment_381.pdf

Harrison D.-H., 2008. A critique of pro-poor tourism. Third World Quarterly, vol. 29, $\mathrm{n}^{\circ}$ 5, p. 851-868.

Hofmann E., Marius-Gnanou K., 2007. Le microcrédit est-il le faux nez du néolibéralisme ? La microfinance et les femmes pauvres : état du débat. Cahiers d'Outre-Mer, $\mathrm{n}^{\circ}$ 238, p. 217-233.

Kabeer N., 2008. Paid Work, Women's Empowerment and Gender Justice: Critical Pathways of Social Change. Pathways Working Paper $n^{\circ} 3$, Institute of Development Studies, Brighton, UK. 121 p. Pathways Working Paper 3. Paid Work, Women's Empowerment and Gender Justice: Critical Pathways of Social Change

Kabeer N., 1999. Resources, agency, achievements: reflections on the measurement of women's empowerment. Development and Change, vol. 30, $\mathrm{n}^{\circ} 3$, p. 435-464. https://www.utsc.utoronto.ca/ $\sim \mathrm{kmacd} / \mathrm{IDSC} 10 /$ Readings/research\%20design/empowerment.pdf

Kaspar H., 2005. "I am the Household Head now!" Gender Aspects of Out-migration for Labour in Nepal. Nepal Institute of Development Studies (NIDS) / Swiss National Centre for Competence in Research (NCCR) North-South, 147 p. http://www.academia.edu/1846026/

I_am_the_Head_of_the_Household_now_Gender_Aspects_of_Out-

Migration_for_Labour_in_Nepal

Khanal M.-N., Shrestha D.-R., Pant P.-D., Mehata S., 2013. Impact of male migration on contraceptive use, unmet need, and fertility in Nepal. Further analysis of the 2011 Nepal demographic and health survey. Calverton, Maryland, Nepal Ministry of Health and Population, New Era and ICF International. https://dhsprogram.com/pubs/pdf/FA74/FA74.pdf

Khanal N.-R., Watanabe T., 2006. Abandonment of Agricultural Land and Its Consequences. A Case Study in the Sikles Area, Gandaki Basin, Nepal Himalaya. Mountain Research and Development, vol. 26, n 1, p. 32-40. http://www.bioone.org/doi/pdf/ 10.1659/0276-4741\%282006\%29026\%5B0032\%3AAOALAI\%5D2.0.CO\%3B2

Lama W.-B., 2000. Community based tourism for conservation and women's development. In P.M. Godde, M.-F. Price, F.-M. Zimmermann (eds), Tourism and Development in Mountain Regions. Oxon and New York: CABI Publishing, p. 221-236.

Létang M., Dérioz P., Le Noac'h J., 2017 (à paraître). Développement touristique, approches patrimoniales et arrangements sociaux en versant sud des Annapurna (Népal). BAGF-Géographies.

Louargant S., Perrin-Bensahel L., 2007. Une approche de la notion de ressources territorialisées par la problématique de genre. In H. Gumuchian, B. Pecqueur (dir.), La ressource territoriale. Anthropos Economica, p 191-208. https://halshs.archives-ouvertes.fr/halshs-00261771/ document 
Louhichi K., 1997. The Impact of the Emigration of the Husband on the Status of the Wife: The Case of Egypt. In M.E. Cosio-Zavala ed., Women and Families: Evolution of the Status of Women as Factor and Consequence of Changes in Family Dynamics. Paris, CICRED, p. 323-339. http://www.cicred.org/ Eng/Publications/Books/Unesco1997/UnescoLouhichi.pdf

Luitel S., 2001. The Social World of Nepalese Women. Occasional Papers in Sociology and Anthropology, vol. 7, p. 101-114. http://himalaya.socanth.cam.ac.uk/collections/journals/opsa/ pdf/OPSA_07_07.pdf

Maharjan A., Bauer S., Knerr B., 2012. Do Rural Women Who Stay Behind Benefit from Male Outmigration? A Case Study in the Hills of Nepal. Gender Technology and Development, vol. 16, $\mathrm{n}^{\circ} 1$, p. 95-123.

Mahat I., 2003. Women's Development in Nepal: The Myh of Empowerment. PRAXIS The Fletcher Journal of International Development, vol. XVIII, p. 67-72. http://fletcher.tufts.edu/ /media/ Fletcher/Microsites/praxis/xviii/Mahat.pdf

Malhotra A., Schüler S., Boender C., 2003. Measuring women's empowerment as a variable in international development. Insight and Action Seminar 12/11/2003, International Council for Research on Women, Washington DC. http://siteresources.worldbank.org/INTEMPOWERMENT/ Resources/486312-1095970750368/529763-1095970803335/malhotra.pdf

Matthiessen P., 2003 ( $1^{\text {st }}$ ed. 1978). The Snow Leopard. Vintage Books, London, 312 p.

Mondain N., Randall S., Diagne A., Elliot A., 2012. Les effets de l'émigration masculine sur les femmes et leur autonomie : entre maintien et transformation des rapports sociaux de sexe traditionnels au Sénégal. Autrepart, $n^{\circ} 61$ (2/2012), p. 81-97. www.cairn.info/revueautrepart-2012-2-page-81.htm

Moser A., 2007. Genre et Indicateurs. Panorama. Bridge Development-Gender, UNDP, Institute of Development Studies, 60 p.http://docs.bridge.ids.ac.uk/vfile/upload/4/document/1105/ Indicators_OR_french.pdf

Mrema A.A., 2014. Tourism and women empowerment in Monduli District, Arusha-Tanzania. African Journal of Hospitality, Tourism and Leisure, vol. 4, $\mathrm{n}^{\circ} 2 . \mathrm{http}: / / w w w . a j h t l . c o m /$ uploads/ 7/1/6/3/7163688/article54rrvol4_2_july-nov2015.pdf

Muzzini E., Aparicio G., 2013. Urban Growth and Spatial Transition in Nepal: An Initial Assessment. World Bank Group e-library, 150 p. http://dx.doi.org/10.1596/978-0-8213-9659-9

Nepal K.S., 2002. Tourism as a key to sustainable mountain development: the Nepalese Himalayas in retrospect. Unasylva, n² 208, vol. 53, p. 38-45. ftp://ftp.fao.org/docrep/fao/004/y3549e/ y3549e03.pdf

Oiry-Varacca M., 2016. Tourisme et politisation des « identités » : réflexions post-coloniales sur la marginalité au Maroc. L'Espace Politique, n² 28 (2016/1). http://espacepolitique.revues.org/3744

Oiry-Varacca M., 2014. Quand le tourisme recompose les identités collectives : étude de projets touristiques « alternatifs » dans les Atlas marocains. Thèse de doctorat en géographie, éd. Faculté des Sciences Economiques et Sociales, Université de Genève, 429 p. https://archive-ouverte.unige.ch/ unige:39568

Pabion Mouriès J., 2012. Tourisme et « lutte contre la pauvreté » au Kirghizstan post-soviétique (Asie centrale) : Vers un éclairage anthropologique. Téoros, vol. 31, n², p. 84-94. http:// teoros.revues.org/2337

Parpart J.S., Rai S.M., Staudt K.A. (eds), 2002. Rethinking Empowerment: gender and development in a global/local world. Routledge, Warwick studies in globalisation (n³), London, 272 p. [en particulier 
“an introduction" (p. 3-21) \& chap. 6, Staudt K.A., Engaging action. Beyond official empowerment discourse (p. 97-110)]

Parpart, J., 2002. Gender and empowerment: new thoughts, new approaches. In V. Desai, R. Potter (eds), The Companion to Development Studies, ed. Edward Arnold, London, p. 338-342.

Pearson R., 2004. Women,Work and Empowerment in a Global Era. IDS Bulletin, vol. 35, nº 4, Repositioning Feminisms in Development, p. 117-120.

Pignède B., 1993. The Gurungs. A Himalayan Population of Nepal. English ed. by S. Harrisson and A. Macfarlane, Ratna Pustak Bhandar, Kathmandu, 523 p. (1 ${ }^{\text {st }}$ ed. Paris, 1966).

Pokharel T., 2010. Gender Equality and Economic Growth of Nepal: A Positive Synergy Hypothesis. Nepal Population Journal, vol. 15, n 14, p. 79-88. http://iussp.org/sites/default/files/ event_call_for_papers/IUSSP\%20Conference_Trilochan\%20Pokharel_0.pdf

Rao A., Kelleher D., 2005. Is there life after gender mainstreaming?. Gender and Development, vol. 13, n 2, p. 57-69. http://yfa.awid.org/wp-content/uploads/2010/03/

life_after_mainstreaming.pdf

Rai S.M., Parpart J.L., Staudt K.A., 2007. (Re)defining empowerment, measuring survival. Workshop on Empowerment: Obstacles, Flaws, Achievements, 3-5 ${ }^{\text {th }}$ May, 2007, Carleton University, Ottawa, Canada. http://www.ethicsofempowerment.org/papers/RaiEmpowerment.pdf Sacareau I. 1999. Les transformations d'une haute montagne par le tourisme : le massif des Annapurna dans l'Himalaya du Népal. Annales de Géographie, n605, Armand Colin, p. 21-45.

Sacareau I., 2009. Changes in environmental policy and mountain tourism in Nepal. Journal of Alpine Research, t. 97, $\mathrm{n}^{\circ}$ 3, p. 1-13.

Scheyvens R., 2000. Promoting Women's Empowerment Through Involvement in Ecotourism: Experiences from the Third World. Journal of sustainable tourism, vol. 8, n 3, p. 232-249. http:// www.tandfonline.com/doi/abs/10.1080/09669580008667360

Sen A., 2009. The idea of justice. Londres, Penguin Books, 558 p.

Shakya K., Gubhaju B., 2016. Factors Contributing to Fertility Decline in Nepal. Journal of Population and Social Studies, vol. 24, n 1, p. 13-29. http://www.jpss.mahidol.ac.th/PDF/JPSSv24n1_Kushum_Bhakta_Fertility_decline_Nepal.pdf

Shakya K., 2014. Changing Gender Status: Achievements and Challenges. In Population Monograph of Nepal, vol. 2 (Social Demography), chap. 6, Central Bureau of Statistics, Kathmandu, p. 221-271. http://cbs.gov.np/image/data/Population/Population Monograph of Nepal 2014/Population Monograph V02.pdf

Sherpa Y., 2007. Mountain Tourism: A Boon or a Bane? Impacts of Tourism on Himalayan Women. International Women of the Moutain, $1^{\text {st }}$ conference, http://www.womenofthemountains.org/files/ Microsoft\%20Word\%20-\%2007-03-01-From-Yankila-Sherpa-FINAL-PAPER-

Final_paper_sent_to_Baktybek.pdf

Singh Dangol B.D., 2014. Fertility Levels, Patterns and Trand. In Population Monograph of Nepal, vol. 1 (Population Dynamics), chap. 5, Central Bureau of Statistics, Kathmandu, p. 115-126. http:// un.org.np/sites/default/files/Population Monograph 2014 Volume1.pdf

Smadja J., 2003. Unités géographiques et paysages au Népal. Terminologies locales. In J. Smadja dir., Histoire et devenir des paysages en Himalaya, chap. 1, coll. Espaces et Milieux, CNRS éd., Paris, p. 51-89. 
Sony K.C., 2012. Roles and Challenges of Women in Tourism Sector of Western Nepal: A MicroEthnographic Study. Nepal Tourism and Development Review, n 2, p. 32-59. http://www.nepjol.info/ nepal/index.php/NTDR/article/view/7379

Stuhaug L. U., 2013. Migration and Rural Livelihoods in a Himalayan Village. The impact of migration on individual households and community development in Nepal. Department of Geography, University of Bergen, 123 p. http://bora.uib.no/bitstream/handle/1956/7287/106812451.pdf?sequence=1

Subedi P., 1997. Nepali women rising. Sahayogi Press, Kathmandu, 145 p.

Swain M., 1989. Gender Roles in Indigenous Tourism: Kuna Mola, Kuna Yala, and Cultural Survival. In V. Smith ed., Hosts and Guests: The Anthropology of Tourism, Philadelphia, University of Pennsylvania Press, p. 83-104.

Thapa Adhikari K., 2013. Do Women Work and Men Decide?Gender Dimensions of Cash Cropping in the Middle Hills of Nepal; Department of Geography, University of Bergen, Norway, 116 p. http:// bora.uib.no/bitstream/handle/1956/7000/106845633.pdf?sequence=1

Thapaliya M., Rai G.S., Shrestha A., Parajuli B., Pande. O., 2012. Home-stay: Assessment in Lwang Ghalel. Nepal Tourism and Development Review, $\mathrm{n}^{\circ}$ 2, p. 105-140. http://www.nepjol.info/nepal/ index.php/NTDR/article/view/7382

Thieme S., 2006. Social Networks and Migration: Far West Nepalese Labour Migrants in Delhi. NCCR North-South dialogue, NCCR North-South, Berne, 61 p. http://www.nccr-north-south.unibe.ch

Thieme S., Müller-Böker U., Backhaus N., 2011. Women's livelihoods in a transnational social space between Nepal and India. In Saraswati R. Ed., Gendered Geographies: Interrogating Place and Space, South Asia Oxford University Press, Delhi, India, p. 60-81.

Thompson A., 2007. The Annapurna Conservation Area Project: tourists as agents of development and environmental management in the high Himalaya? International Journal of Environment and Sustainable Development, vol. 6, $\mathrm{n}^{\circ}$ 4, p. 405-421.

Tiekoura O., 2012. Tourisme et identités de genre à Agadez, Niger. Via@, Tourismes et dynamiques identitaires, $\mathrm{n}^{\circ}$ 2. http://viatourismreview.com/wp-content/uploads/2015/06/Article13.pdf

Tizza W., 2012. Femmes des Aït Bouguemez : des identités à l'épreuve du tourisme.Via@, Tourismes et dynamiques identitaires, $\mathrm{n}^{\circ} 2$. http://viatourismreview.com/wp-content/uploads/ 2015/06/Article12.pdf

Upadhayaya P.K., 2013. Peace Sensitivity in Tourism codes of Conduct at Destination in Conflict: A Case Study of Partnership Actions in Pokhara, Western Nepal. In Wohlmuther C. \&

Wintersteiner W. eds., International Handbook on Tourism and Peace. Klagenfurt, Drava. p. 230-246. http://www.uni-klu.ac.at/frieden/downloads/

International_Handbook_on_Tourism_and_Peace.pdf

Upadhayaya P.K., 2011. Peace through tourism. A critical look at Nepalese Tourism. Nepal Tourism and Development Review, vol. 1, n 1, p. 15-40. http://www.nepjol.info/index.php/NTDR/article/ view/7368

Upadhayaya P.K., Upreti B.R., 2008. Enhancing women's participation in mountain tourism, prospects and challenges. http://lib.icimod.org/record/13555/files/1265.pdf

Yabiku S.T., Agadjanian V., Sevoyan A., 2010. Husbands' Labour Migration and Wives' Autonomy. Population Studies, vol. 64, $n^{\circ}$ 3, p. 293-306. http://www.ncbi.nlm.nih.gov/pmc/articles/PMC2976678/ 
Zahedi N., 2010. Women in tourism, Opportunity or Impediment? BA Thesis, Institute of Human Geography, Planning and International Development Studies, University of Amsterdam, 35 p. http://dare.uva.nl/cgi/arno/show.cgi?fid=352294

\section{NOTES}

1. Source: Nepal National Census - 2011. La population «absente» correspond dans le recensement à la population absente du foyer pendant une période de 6 mois ou davantage. En 2011 , plus d'un quart $(25,4 \%)$ des foyers népalais déclaraient que l'un de ses membres vivait à l'étranger, et le nombre des « absents » (1,92 millions de personnes) avait plus que doublé depuis 2001 (760 000 personnes).

2. $72 \%$ des hommes ont migré à la recherche d'un emploi, alors que les migrations des femmes sont dues avant tout au mariage ( $54 \%$ ) ou à des « raisons familiales » (22\%), qui incluent le fait de se rapprocher de leur conjoint ou de leurs enfants (Source : Nepal National Census - 2011).

3. $25,7 \%$ en moyenne, pour une situation un peu plus fréquente en milieu urbain $(28,1 \%)$ qu'en zone rurale $(25,2 \%)$.

4. “Tourism and hotels have increased women's engagement in trade and related services, like roadside tea stalls, village level guesthouses, pubs etc., which fall under the multiple economic activities as included in the definition of economic activities. Women in Nepal have always managed shops, but previously they would have declared themselves as housewives" (Shakya, 2014, p. 258).

5. « A qui appartiennent les paysages en Asie ?», 2014-2017, direction E. Gauché (UMR CITERES), université de Tours). Inscrit dans une perspective comparative, le programme AQAPA se déploie sur cinq terrains d'études dans cinq pays différents, Inde (Kumaon-Uttarakhand), Chine (Guizhou), Vietnam (Lam Dong), Laos (Louang Namtha) et Népal. (https:// aqapa.hypotheses.org/).

6. Mélanie Pauget (Master tourisme ISTHIA, avril-juin 2015) et Justine Le Noac'h (Master Géoter Université d'Avignon, avril-mai 2016).

7. Les entretiens se sont déroulés tantôt en anglais, tantôt en nepali avec l'entremise d'un traducteur, qui était souvent P.K. Upadhayaya (Université de Kathmandu), correspondant népalais pour le programme AQAPA et membre de l'équipe.

8. Créé en 1986, l'ACAP correspond au plus ancien, au plus vaste et au plus touristique des périmètres protégés du Népal. Il est aujourd'hui géré par une ONG jouant le rôle d'une administration d'État, le National Trust for Nature Conservation (NTNC) (Sacareau, 1999 et 2009 ; Thomson, 2007), et tire une partie importante de ses ressources des droits d'entrée versés par les touristes étrangers.

9. VDC : Village Development Committee, niveau élémentaire de l'administration et de la gestion territoriale au Népal Le sigle renvoie à la fois au périmètre concerné et au comité qui l'administre. Les VDC sont généralement composés de neuf wards, qui sont des subdivisions électorales internes.

10. «Il n'y a plus de routes à l'ouest de Pokhara, qui est le dernier avant-poste du monde moderne. En une journée de marche, on se trouve à un siècle de distance ».

11. 5 à 7 jours de marche pour le Balcon des Annapurnas, 8 à 10 jours pour le Sanctuaire des Annapurna (ABC), l'un et l'autre accessibles à des trekkeurs individuels.

12. L'expérience du Machhapuchhre Model Trek (MMT) a été développée en 2009-2010 sur le territoire de sept VDC, à l'initiative conjointe du NCCR North-South (Swiss National Centre of Competence in Research), de TAAN Pokhara (Trekking Agencies' Association of Nepal / Western Regional Chapter), et de l'Université de Kathmandu (Human and Natural Resources Studies Centre). 
13. Les ordres de grandeur pour 2013 étaient de l'ordre de 113000 touristes étrangers pour l'ensemble de l'ACAP, dont 52000 sont passés par Ghandruk, en route vers l'ABC (source ACAP). Ghandruk a également attiré une importante clientèle népalaise, plus difficile à évaluer car les Népalais n'ont pas besoin de permis pour entrer dans l'ACAP, mais l'ACAP l'évaluait autour de 80000 personnes pour 2013. En comparaison, le trek du Mardi Himal ne représente encore que 1500 à 2000 personnes par an, et la fréquentation des homestays de Lwang restait en 2013 légèrement inférieure à 700 touristes, dont trois quarts de Népalais (source ACAP).

14. Les touristes internationaux qui fréquentent ces homestays sont pour la plupart des voyageurs avertis, à la recherche d'un contact plus direct avec les sociétés locales que celui qu'autorisent les formules classiques du trekking.

15. Autour de $40 \%$ des ménages ayant au moins un membre à l'étranger.

16. Nombre de ménages recevaient également de l'argent de membres ayant migré à l'intérieur du Népal, notamment vers Pokhara ou Kathmandu, mais il s'agit généralement là de migrations définitives, et non de " population absente ».

17. L'Inde reste le pays de destination de $37,5 \%$ des migrants, mais ce taux, qui dépassait les $90 \%$ dans les années 1980, a beaucoup diminué (source : Nepal National Census-2011). Le recul est allé s'accélérant depuis que les recensements intègrent cette information : $93 \%$ en 1981, $89 \%$ en 1991, 77,3 \% en 2001. Les pays du Golfe (Arabie Saoudite, Qatar, Émirats Arabes Unis...) accueillent dorénavant un nombre de migrants népalais équivalent à celui de l'Inde ; parmi les destinations est-asiatiques, la Malaisie a également pris une place de premier plan depuis la fin des années 2000 .

18. De 5,1 enfants par femme en moyenne en 1991 à 2,6 en 2011.

19. Western Development Region, Kaski District, dans la zone des collines au sud-ouest de Pokhara.

20. Il peut toutefois se produire, comme nous l'avons observé pour l'un des homestays du village de Lwang, que le foyer ainsi reconstitué soit malgré tout exclusivement féminin, parce que le beau-père travaille également à l'étranger.

21. Trek du Balcon des Annapurna, VDC de Ghandruk. Ulleri est un village Poon Magar. Ghorepani, à la population plus mélangée, appartient déjà au district voisin de Myagdi.

22. Entretien 09/05/2015.

23. Entretien 09/05/2015.

24. Pour ces 55 VDC, la décision s'opère au sein d'un comité élargi (Conservation and Development Committee) de 15 membres incluant les 9 membres initiaux du VDC auxquels s'ajoutent 6 membres sélectionnés par l'ACAP de manière à ce que ce CDC comporte au moins deux femmes, un travailleur social et un représentant d'un groupe défavorisé (intouchables) (Gurung, 2004, p. 27-29)

25. Il s'agit de la Trekkers Inn : http://www.hoteltrekkersinnghandruk.com/

26. Pour l'ensemble des 11 VDC étudiés, les hautes-castes indo-népalaises représentent un tiers de la population (Brahman des collines $2 \%$, Chetri $7 \%$, Thakuri $1 \%$ ), faisant à peu près jeu égal avec les ethnies tibeto-birmanes (Gurung $19 \%$, Magar $10 \%$, Tamang $5 \%$ ). Les populations indonépalaises de basses castes et les intouchables (Kami $16 \%$, Sarki $7 \%$, Damai-Dholi $6 \%$, autres Dalits $1,5 \%$ ) composent à peu de choses près le dernier tiers, même si en tout sont recensées une vingtaine de castes et d'ethnies différentes. Mais la composition varie beaucoup d'un village à l'autre, par exemple entre Lahachok, village indo-népalais dominé par les Bahun-Chetri à $58 \%$, Gandhruk, VDC Gurung à $42 \%$, et Dangsing, où aucun groupe ne domine fortement.

27. Terme qui désigne la situation de personnes subissant simultanément plusieurs formes de domination ou de discrimination dans une société. L'intersectionnalité étudie les formes de domination et de discrimination non pas séparément, mais dans les liens qui se nouent entre elles.

28. Source ACAP 


\section{RÉSUMÉS}

Essentiellement masculine, l'émigration internationale de travail constitue au Népal, un phénomène ancien et de grande ampleur. Dans les régions où il est le plus actif, il se traduit par une nette féminisation des sociétés locales, qui ne s'accompagne pas partout d'un gain en autonomie et en indépendance pour les femmes qui restent. A partir d'une étude de cas en versant sud du massif des Annapurna où l'absence des hommes est particulièrement marquée, cet article tente de préciser la manière dont le développement touristique, en tant que secteur d'activité, offre des opportunités de travail, et au-delà d'émancipation, aux femmes qui sont en mesure de s'en saisir.

Predominantly male, the labor out-migration is in Nepal a large-scale phenomenon which has a long history. In the areas where it is the most important, this process leads to a clear feminization of the local societies. But it don't mean in every case that the womens who stay behind have win something regarding to their autonomy and independance. This paper is based on a case study on the southern slope of the massif of Annapurna, in a tourist area where the absence of the men is particularly marked. Its pupose is to analyze in what extend the development of the tourist sector offers working opportunities - and at the same time opportunities of empowerment - to the womens who are in capacity to seize them.

\section{INDEX}

Mots-clés : émigration de travail, développement touristique, sex-ratio, rapport de genre, empowerment, Himalaya népalais

Keywords : labour out-migration, tourism development, gender relationships, empowerment, nepalese Himalaya

Thèmes : Sur le Champ - Sur le Terrain

\section{AUTEURS}

\section{PIERRE DÉRIOZ}

Pierre Dérioz, pierre.derioz@univ-avignon.fr, Géographe, est Maître de conférences HDR à l'Université d'Avignon et des Pays de Vaucluse, membre de l'UMR Espace-Dev 228 IRD / OHM Pyrénées - Haut Vicdessos. Il a publié récemment :

- Dérioz P., Bachimon P., Loireau M., 2015. Mise en scène du paysage montagnard et valorisation sélective des patrimoines dans une vallée pyrénéenne en reconversion économique (Vicdessos, Ariège). Projets de paysage, $\mathrm{n}^{\circ} 11$, articles issus du colloque « Paysage(s) et Patrimoine(s) : connaissance, reconnaissance, protection, gestion et valorisation », ENSPN Blois, 24-25 septembre 2013. http://www.projetsdepaysage.fr/ mise_en_scene_du_paysage_montagnard_et_valorisation_selective_des_patrimoines_dans_une_vallee_pyreneenne_en_reconversion_ - Bachimon P., Dérioz P., Vlès V., 2016. Le dédoublement résidentiel, descripteur des bifurcations des trajectoires des stations de montagne. In V. Vlès et C. Bouneau (dir.), Stations en tension, partie 1, chap. 2, P.I.E. Peter Lang, Bruxelles, p. 31-48 (bibliographie en fin d'ouvrage) - Dérioz P., Bachimon P., Loireau M., Upadhayaya P.K., Arcuset L., 2017, à paraître. Systèmes touristiques territorialisés, degré de spécialisation fonctionnelle des territoires et coviabilité : 
approche générale et enseignements de quelques études de cas. In O. Barrière, S. Morand, M. Behnassi, G. David, V. Douzal, V.R. Canete, C. Prost, T. Libourel, M. Fargette, M. Loireau, L. Pascal, F. Seyler (edts), Co-viability of Social and Ecological Systems: Reconnect Man to the Biosphere in a Global Era of Change, Partie 3 : La coviabilité interrogée par la diversité des situations, ed. Springer.

\section{PRANIL UPADHAYAYA}

Pranil Upadhayaya, pranilupadhayaya@gmail.com, est Enseignant en Tourisme et chercheur associé à l'Université de Kathmandu (Népal), membre du Nepal Center for Contemporary Research (NCCR) / OHM Pyrénées - Haut Vicdessos. Il a publié récemment :

- Upadhayaya P.K., 2014. Transdiciplinary Research as the Peace Prospective for Community Based Village Tourism: An Example of Machhapuchhre Model Trek in Western Nepal. SHS Web of conferences 12, EDP Science ; http://www.shs-conferences.org/articles/shsconf/pdf/2014/09/ shsconf_4ictr2014_01038.pdf

- Dérioz P., Bachimon P., Loireau M., Upadhayaya P.K., Arcuset L., 2017, à paraître. Systèmes touristiques territorialisés, degré de spécialisation fonctionnelle des territoires et coviabilité : approche générale et enseignements de quelques études de cas. In O. Barrière, S. Morand, M. Behnassi, G. David, V. Douzal, V.R. Canete, C. Prost, T. Libourel, M. Fargette, M. Loireau, L. Pascal, F. Seyler (edts), Co-viability of Social and Ecological Systems: Reconnect Man to the Biosphere in a Global Era of Change, Partie $3:$ La coviabilité interrogée par la diversité des situations, ed. Springer.

\section{MAUD LOIREAU}

Maud Loireau, maud.loireau@ird.fr, Géo-agronome, est Ingénieur de recherches à l'UMR EspaceDev 228 IRD (Montpellier)/OHM Pyrénées - Haut Vicdessos. Elle a publié récemment :

- - Dérioz P., Bachimon P., Loireau M., 2015. Mise en scène du paysage montagnard et valorisation sélective des patrimoines dans une vallée pyrénéenne en reconversion économique (Vicdessos, Ariège). Projets de paysage, $\mathrm{n}^{\circ} 11$, articles issus du colloque « Paysage(s) et Patrimoine(s) : connaissance, reconnaissance, protection, gestion et valorisation », ENSPN Blois, 24-25 septembre 2013. http://www.projetsdepaysage.fr/

mise_en_scene_du_paysage_montagnard_et_valorisation_selective_des_patrimoines_dans_une_vallee_pyreneenne_en_reconversion_ - Dérioz P., Bachimon P., Loireau M., Upadhayaya P.K., Arcuset L., 2017, à paraître. Systèmes touristiques territorialisés, degré de spécialisation fonctionnelle des territoires et coviabilité : approche générale et enseignements de quelques études de cas. In O. Barrière, S. Morand, $\mathrm{M}$. Behnassi, G. David, V. Douzal, V.R. Canete, C. Prost, T. Libourel, M. Fargette, M. Loireau, L. Pascal, F. Seyler (edts), Co-viability of Social and Ecological Systems: Reconnect Man to the Biosphere in a Global Era of Change, Partie $3:$ La coviabilité interrogée par la diversité des situations, ed. Springer.

\section{PHILIPPE BACHIMON}

Philippe Bachimon, philippe.bachimon@neuf.fr, Géographe, est Professeur à l'Université d'Avignon et des Pays de Vaucluse, UMR Espace-Dev 228 IRD / OHM Pyrénées - Haut Vicdessos. Il a publié récemment :

- Dérioz P., Bachimon P., Loireau M., 2015. Mise en scène du paysage montagnard et valorisation sélective des patrimoines dans une vallée pyrénéenne en reconversion économique (Vicdessos, Ariège). Projets de paysage, $\mathrm{n}^{\circ} 11$, articles issus du colloque « Paysage(s) et Patrimoine(s) : connaissance, reconnaissance, protection, gestion et valorisation », ENSPN Blois, 24-25 septembre 2013. http://www.projetsdepaysage.fr/ mise_en_scene_du_paysage_montagnard_et_valorisation_selective_des_patrimoines_dans_une_vallee_pyreneenne_en_reconversion_ - Bachimon P., Dérioz P., Vlès V., 2016. Le dédoublement résidentiel, descripteur des bifurcations des trajectoires des stations de montagne. In V. Vlès et C. Bouneau (dir.), Stations en tension, 
partie 1, chap. 2, P.I.E. Peter Lang, Bruxelles, p. 31-48 (bibliographie en fin d'ouvrage).

- Dérioz P., Bachimon P., Loireau M., Upadhayaya P.K., Arcuset L., 2017, à paraître. Systèmes touristiques territorialisés, degré de spécialisation fonctionnelle des territoires et coviabilité : approche générale et enseignements de quelques études de cas. In O. Barrière, S. Morand, M. Behnassi, G. David, V. Douzal, V.R. Canete, C. Prost, T. Libourel, M. Fargette, M. Loireau, L. Pascal, F. Seyler (edts), Co-viability of Social and Ecological Systems: Reconnect Man to the Biosphere in a Global Era of Change, Partie 3 : La coviabilité interrogée par la diversité des situations, ed. Springer.

\section{JUSTINE LE NOAC'H}

Justine Le Noac'h, justinelenoach@gmail.com, est Étudiante en Master « Géomatique et projets territoriaux », Université d'Avignon et des pays de Vaucluse.

\section{MAUVE LÉTANG}

Mauve Létang, mauveletang1@gmail.com, est Doctorante en Géographie, Université ParisSorbonne, UMR ENeC et conduit ses recherches notamment dans le cadre du programme ANR AQAPA : http://aqapa.hypotheses.org/ et du programme SYSTERPA, labellisé par l'Observatoire Hommes-Milieux du Haut-Vicdessos : http://w3.ohmpyr.univ-tlse2.fr/programme2013_6.php Labex DRIIHM. Elle a publié récemment :

- Létang M., 2017, soumis. Gouvernements de l'environnement et circulation des inégalités dans le village de Sarmoli (Kumaon, Uttarakhand Himalaya indien). Une lecture par le prisme du capital environnemental, Développement durable et Territoires. 